\title{
Synthesis of the $C(43)-C(67)$ Fragment of Amphidinol 3
}

\author{
Jacqueline D. Hicks, ${ }^{a}$ Eric M. Flamme, ${ }^{a}$ and William R. Roush ${ }^{* b}$ \\ aDepartment of Chemistry, University of Michigan, Ann Arbor, MI 48109 and \\ ${ }^{\mathrm{b}}$ Department of Medicinal Chemistry, Scripps-Florida, Jupiter, FL 33435 \\ Email: roush@scripps.edu
}

Supporting Information

Part 1-Experimental Procedures 
General Methods: All reaction solvents were purified before use. $\mathrm{CH}_{2} \mathrm{Cl}_{2}, \mathrm{Et}_{2} \mathrm{O}$, toluene, and THF were purified by passage through a solvent column composed of activated alumina (A-1). Unless otherwise indicated, all chemicals were used as purchased with out further purification. All reactions were conducted under a dry nitrogen atmosphere using oven dried $\left(140^{\circ} \mathrm{C}\right)$ glassware. Removal of solvents was accomplished on a rotary evaporator at reduced pressure. Absolute configurations were determined using the modified Mosher method. ${ }^{1}$

Physical Properties and Spectroscopic Measurements: ${ }^{1} \mathrm{H}$ NMR spectra were recorded on a Varian VXR-400 spectrometer at $400 \mathrm{MHz}$ or on a Varian Inova-500 spectrometer at $500 \mathrm{MHz} .{ }^{13} \mathrm{C} \mathrm{NMR}$ spectra were recorded on a Varian VXR-400 spectrometer at $100 \mathrm{MHz}$ or a Varian Inova-500 spectrometer at $125 \mathrm{MHz}$. The proton signal of residual, non-deuterated solvent $\left(\square 7.27 \mathrm{ppm}\right.$ for $\left.\mathrm{CHCl}_{3}\right)$ was used as an internal reference for ${ }^{1} \mathrm{H}$ spectra. For ${ }^{13} \mathrm{C}$ spectra, the chemical shifts are reported relative to the $\square 77.0 \mathrm{ppm}$ resonance of $\mathrm{CDCl}_{3}$. Coupling constants are reported in Hertz $(\mathrm{Hz})$. Infrared (IR) spectra were recorded as thin films using $\mathrm{CHCl}_{3}$ as the solvent on a Perkin-Elmer Spectrum $1000 \mathrm{FTIR}$. Optical rotations were measured on a Rudolph Autopol III polarimeter using a quartz cell with $1 \mathrm{~mL}$ capacity and a $10 \mathrm{~cm}$ path length. Mass spectra were recorded on a VG 70-250-s spectrometer manufactured by Micromass Corp. (Manchester UK) at the University of Michigan Mass Spectrometry Laboratory.

Analytical thin layer chromatography (TLC) was performed on Kieselgel 60 F254 glass plates precoated with a $0.25 \mathrm{~mm}$ thickness of silica gel. TLC plates were visualized with UV light and/or by

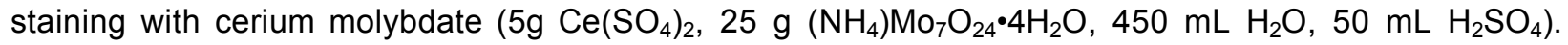
Column chromatography was generally performed according to the method of Still using Kieselgel 60 (230-400 mesh) silica gel. ${ }^{2}$

HPLC purifications were performed by using an HPLC system composed of two Rainin HPLC pumps connected to a Dynamax axial compression column packed with Rainin $60 \AA$ irregular silica gel. Samples were loaded into the system with a $2 \mathrm{~mL}$ Rheodyne 7125 injector and were detected by using a Rainin Dynamax UV-C detector.

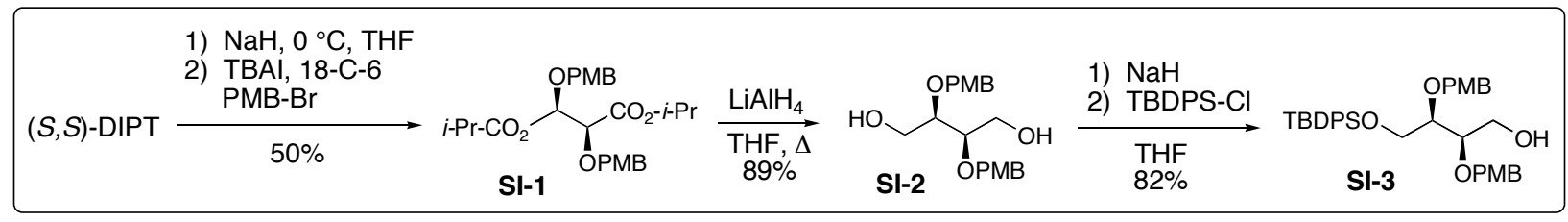

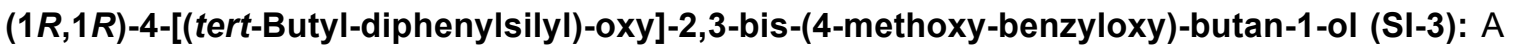
solution of diisopropyl $(S, S)$-tartrate $(1.00 \mathrm{~g}, 4.27 \mathrm{mmol})$ in THF $(6 \mathrm{~mL})$ was cooled in an ice bath. In a separate flask $\mathrm{NaH}(0.326 \mathrm{~g}, 8.15 \mathrm{mmol}, 60 \%$ dispersion in oil) was suspended in $6 \mathrm{~mL}$ of THF and the suspension was cooled in an ice bath. The tartrate solution was then added dropwise over 30 min to the flask containing the $\mathrm{NaH}$ suspension and the reaction was stirred for $1 \mathrm{~h}$. Tetrabutylammonium iodide 
$(0.315 \mathrm{~g}, 0.854 \mathrm{mmol})$ was then added followed by 18 -crown-6 $(2.5 \mathrm{mg}, 9.4 \mathrm{mmol})$. p-Methoxybenzyl bromide $(1.64 \mathrm{~g}, 8.15 \mathrm{mmol})$ was then added dropwise to the mixture over $30 \mathrm{~min}$. The cold bath was removed and the reaction was allowed to stir for $1 \mathrm{~h}$ at ambient temperature. The reaction mixture was diluted with $1 \mathrm{M} \mathrm{HCl}, \mathrm{Et}_{2} \mathrm{O}$ and $\mathrm{H}_{2} \mathrm{O}$. The aqueous layer was extracted with $\mathrm{Et}_{2} \mathrm{O}$ and the combined organics were washed with sat. $\mathrm{NaHCO}_{3}(1 \mathrm{x})$, brine (1x), dried over $\mathrm{Na}_{2} \mathrm{SO}_{4}$, and filtered. The solvent was removed in vacuo and the crude oil was purified by column chromatography (85:15, hexanes-EtOAc) to afford $\mathbf{S I}-1$ as a clear oil $(2 \mathrm{~g}, 50 \%)$.

To a $0{ }^{\circ} \mathrm{C}$ suspension of $\mathrm{LiAlH}_{4}(0.16 \mathrm{~g}, 4.3 \mathrm{mmol})$ in $10 \mathrm{~mL}$ of THF was added a solution of the crude diester $\mathbf{S I}-1$ (1.0 g, $2.1 \mathrm{mmol}$ ) in $20 \mathrm{~mL}$ of THF via cannula over $30 \mathrm{~min}$. The reaction flask was removed from the cold bath, fitted with a condenser, and heated at reflux for $3 \mathrm{~h}$. The reaction mixture was then cooled to ambient temperature and solid $\mathrm{Na}_{2} \mathrm{SO}_{4} \cdot 10 \mathrm{H}_{2} \mathrm{O}(2.75 \mathrm{~g}, 8.56 \mathrm{mmol})$ was carefully added and stirred for $1 \mathrm{~h}$. The resulting suspension was vacuum filtered through Celite using a coarse fritted funnel. The THF was removed in vacuo and the remaining residue was dissolved in EtOAc, dried over $\mathrm{Na}_{2} \mathrm{SO}_{4}$, filtered, and concentrated in vacuo to give diol $\mathbf{S I - 2}$ as a colorless oil $(0.69 \mathrm{~g}, 89 \%)$.

$\mathrm{NaH}(75 \mathrm{mg}, 1.8 \mathrm{mmol})$ was suspended in THF $(2 \mathrm{~mL})$ and cooled in an ice bath. A solution of SI-2 $(0.683 \mathrm{~g}, 1.88 \mathrm{mmol})$ in THF $(2 \mathrm{~mL})$ was then added dropwise via cannula. The cold bath was removed and the reaction stirred $1 \mathrm{~h}$ at ambient temperature. To this mixture was added TBDPS-Cl $(0.48$ $\mathrm{mL}, 1.88 \mathrm{mmol}$ ) dropwise and the reaction was stirred for $2 \mathrm{~h}$ before $10 \%$ aq. $\mathrm{K}_{2} \mathrm{CO}_{3}$ solution was added. The aqueous layer was extracted with EtOAc and the combined organics were washed with sat. $\mathrm{NaHCO}_{3}$ (1x), brine (1x), dried, and filtered. The solvent was removed in vacuo and the crude oil was purified by column chromatography $\left(70: 30\right.$, hexanes-EtOAc) to afford alcohol SI-3 as a clear oil $(0.88 \mathrm{~g}, 82 \%)$ : [C] ${ }_{\mathrm{D}}{ }^{26}$ $=-11.8^{\circ}\left(\mathrm{c}=1.08, \mathrm{CHCl}_{3}\right) ;{ }^{1} \mathrm{H}$ NMR $\left(400 \mathrm{MHz}, \mathrm{CDCl}_{3}\right) \square 7.70-7.64(\mathrm{~m}, 4 \mathrm{H}), 7.46-7.34(\mathrm{~m}, 6 \mathrm{H}), 7.20-7.15$ $(\mathrm{m}, 4 \mathrm{H}), 6.86-6.80(\mathrm{~m}, 4 \mathrm{H}), 4.58-4.50(\mathrm{~m}, 3 \mathrm{H}), 4.41$ (B of $\mathrm{ABq}, \mathrm{J}=14.5 \mathrm{~Hz}, 1 \mathrm{H}), 3.88-3.80(\mathrm{~m}, 2 \mathrm{H}), 3.79(\mathrm{~s}$, $3 \mathrm{H}), 3.78(\mathrm{~s}, 3 \mathrm{H}), 3.75-3.58(\mathrm{~m}, 4 \mathrm{H}), 2.11(\mathrm{dd}, \mathrm{OH}, \mathrm{J}=6,8.5 \mathrm{~Hz}, 1 \mathrm{H}), 1.06(\mathrm{~s}, 9 \mathrm{H}) ;{ }^{13} \mathrm{C} \mathrm{NMR}(125 \mathrm{MHz}$, $\left.\mathrm{CDCl}_{3}\right) \square 159.4,159.4,135.9,135.8,133.4,133.3,130.7,130.67,129.98,129.97,129.8,129.8,128.0$, 127.9, 114.0, 113.9, 79.7, 78.7, 72.8, 72.6, 63.1, 62.0, 55.4, 27.0, 19.4; IR (thin film) 3460, 2931, 1612, $1513,1248 \mathrm{~cm}^{-1}$; HRMS (ESI) calcd for $\mathrm{C}_{36} \mathrm{H}_{44} \mathrm{O}_{6} \mathrm{SiNa}(\mathrm{M}+\mathrm{Na})^{+} 623.2805$, found $623.2816 \mathrm{~m} / \mathrm{z}$.

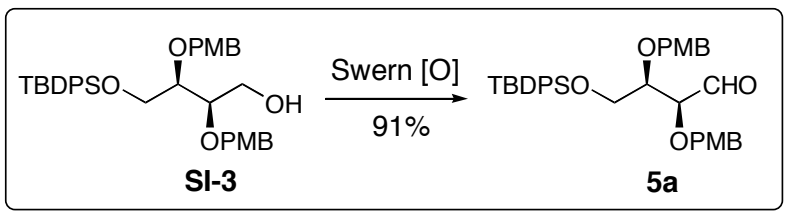

(2S,3R)4-[(tert-Butyl-diphenylsilyl)-oxy]-2,3-bis-(4-methoxy-benzyloxy)-butyraldehyde (5a). Freshly distilled oxalyl chloride (14 $\mathrm{GL}, 0.16 \mathrm{mmol}$, distilled from $\mathrm{CaH}_{2}$ ) was added to $1 \mathrm{~mL}$ of $\mathrm{CH}_{2} \mathrm{Cl}_{2}$ and the solution was cooled to $-78{ }^{\circ} \mathrm{C}$ in a dry ice-acetone bath. Dimethyl sulfoxide ( $23 \mathrm{LL}, 0.32 \mathrm{mmol}$ ) was added dropwise and the mixture was stirred for $20 \mathrm{~min}$. A mixture of alcohol SI-3 (65 mg, $0.11 \mathrm{mmol})$ and $\mathrm{Et}_{3} \mathrm{~N}(90 \mathrm{~mL}, 0.65 \mathrm{mmol})$ in $1 \mathrm{~mL}$ of $\mathrm{CH}_{2} \mathrm{Cl}_{2}$ was added dropwise to the activated DMSO complex solution. 
The reaction mixture was allowed to warm to $0{ }^{\circ} \mathrm{C}$ over $2 \mathrm{~h}$ and then was diluted with sat. $\mathrm{NH}_{4} \mathrm{Cl}$. The aqueous layer was extracted with $\mathrm{CH}_{2} \mathrm{Cl}_{2}$ and the combined organics were washed with $1 \mathrm{M} \mathrm{KHSO}_{4}$, brine $(2 \mathrm{x})$, dried over $\mathrm{Na}_{2} \mathrm{SO}_{4}$, and filtered. The solvent was removed in vacuo and the crude oil was purified by column chromatography (90:10, hexanes-EtOAc) to afford aldehyde 5 a as a clear oil (59 $\mathrm{mg}, 91 \%)$ : $[\square]_{\mathrm{D}}{ }^{25}=-30^{\circ}\left(\mathrm{c}=0.98, \mathrm{CHCl}_{3}\right) ;{ }^{1} \mathrm{H}$ NMR $\left(500 \mathrm{MHz}, \mathrm{CDCl}_{3}\right) \square 9.71(\mathrm{~s}, 1 \mathrm{H}), 7.69-7.62(\mathrm{~m}, 4 \mathrm{H}), 7.48-7.37($ $\mathrm{m}, 6 \mathrm{H}), 7.27-7.23(\mathrm{~m}, 2 \mathrm{H}), 7.12-7.08(\mathrm{~m}, 2 \mathrm{H}), 6.88-6.80(\mathrm{~m}, 4 \mathrm{H}), 4.66(\mathrm{~A}$ of $\mathrm{ABq}, J=11.5 \mathrm{~Hz}, 1 \mathrm{H})$, $4.54(\mathrm{~B}$ of $\mathrm{ABq}, J=11.5 \mathrm{~Hz}, 1 \mathrm{H}), 4.41(\mathrm{~A}$ of $\mathrm{ABq}, J=11.5 \mathrm{~Hz}, 1 \mathrm{H}), 4.30(\mathrm{~B}$ of $\mathrm{ABq}, J=11.5 \mathrm{~Hz}, 1 \mathrm{H})$, $4.04(\mathrm{dd}, J=1.5,3.5 \mathrm{~Hz}, 1 \mathrm{H}), 3.82-3.72(\mathrm{~m}, 9 \mathrm{H}), 1.01(\mathrm{~s}, 9 \mathrm{H}) ;{ }^{13} \mathrm{C}$ NMR $\left(125 \mathrm{MHz}, \mathrm{CDCl}_{3}\right) \mathrm{d} 203.8$, 159.8, 159.6, 135.9, 135.8, 133.3, 130.2, 130.1, 130.0, 129.9, 129.6, 128.0, 128.0, 114.1, 114.0, 82.7, 79.2, 73.5, 72.7, 61.7, 55.5, 27.0, 19.4; IR (thin film) 2932, 1731, 1613, 1514, $1249 \mathrm{~cm}^{-1}$; HRMS (ESI) calcd for $\mathrm{C}_{36} \mathrm{H}_{42} \mathrm{O}_{6} \mathrm{SiNa}(\mathrm{M}+\mathrm{Na})^{+} 621.2648$, found $621.2654 \mathrm{~m} / \mathrm{z}$.

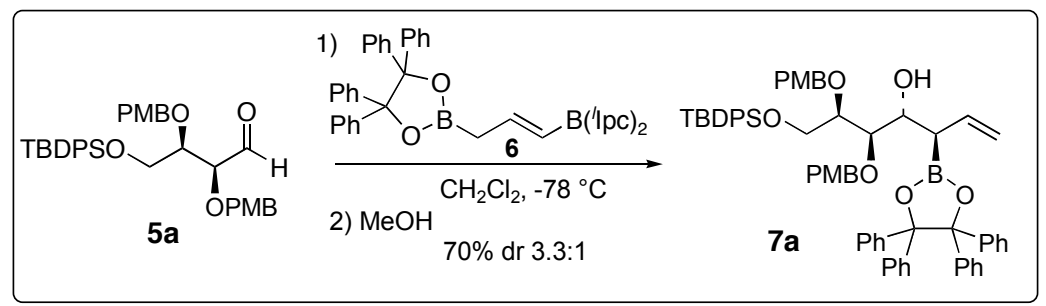

$(3 R, 4 R, 5 R, 6 R)-7$-(tert-Butyl-diphenylsilanyloxy)-5,6-bis-(4-methoxybenzyloxy)-3-(4,4,5,5-

tetraphenyl-[1,3,2]dioxaborolan-2-yl)-hept-1-en-4-ol (7a). 2-Allenyl-4,4,5,5-tetraphenyl-1,3,2dioxaborolane ${ }^{3}(94 \mathrm{mg}, 0.23 \mathrm{mmol})$ and $(' \mathrm{lpc})_{2} \mathrm{BH}^{4}(65 \mathrm{mg}, 0.23 \mathrm{mmol})$ were weighed out in a glove box. The crystalline (' $\mathrm{Ipc})_{2} \mathrm{BH}$ was ground with glass rod (to improve the rate of dissolution) then removed from the glove box and dissolved in $\mathrm{CH}_{2} \mathrm{Cl}_{2}(1.5 \mathrm{~mL})$. The solution was cooled to $0{ }^{\circ} \mathrm{C}$ and a solution of the above allene in $\mathrm{CH}_{2} \mathrm{Cl}_{2}(1 \mathrm{~mL})$ was added dropwise via cannula. The mixture was stirred for $2 \mathrm{~h}$ at $0{ }^{\circ} \mathrm{C}$, then cooled to $-78{ }^{\circ} \mathrm{C}$ and a solution of aldehyde $5 \mathrm{a}(119 \mathrm{mg}, 0.2 \mathrm{mmol})$ in $\mathrm{CH}_{2} \mathrm{Cl}_{2} \quad(0.5 \mathrm{~mL})$ was added dropwise. The reaction mixture was stirred for $2.5 \mathrm{~h}$ at $-78{ }^{\circ} \mathrm{C}$, then $\mathrm{MeOH}(1 \mathrm{~mL})$ was added and the solution was allowed to warm to ambient temperature and stirred for $1.5 \mathrm{~h}$. The reaction mixture was diluted with $\mathrm{CH}_{2} \mathrm{Cl}_{2}(60 \mathrm{~mL})$ and brine and the aqueous layer was extracted with $\mathrm{CH}_{2} \mathrm{Cl}_{2}(2 \mathrm{x})$. The combined organic extracts were dried over $\mathrm{Na}_{2} \mathrm{SO}_{4}$, filtered and concentrated in vacuo. The resulting oil was purified by column chromatography to afford a mixture of allylboronates as a white foam (140 mg, $70 \%$, dr $3.3: 1)$. An analytical sample of 7 a was obtained by preparative HPLC (80:20 hexanes-EtOAc): $[\square]_{\mathrm{D}}{ }^{25}=+19^{\circ}\left(\mathrm{c}=1.08, \mathrm{CHCl}_{3}\right) ;{ }^{1} \mathrm{H}$ NMR $\left(500 \mathrm{MHz}, \mathrm{CDCl}_{3}\right) \square 7.69-7.67(\mathrm{~m}, 4 \mathrm{H}), 7.46-7.41(\mathrm{~m}, 2 \mathrm{H}), 7.39-$ $7.33(\mathrm{~m}, 4 \mathrm{H}), 7.21-7.16(\mathrm{~m}, 7 \mathrm{H}), 7.14-7.09(\mathrm{~m}, 6 \mathrm{H}), 7.08-6.98(\mathrm{~m}, 11 \mathrm{H}), 6.81-6.72(\mathrm{~m}, 4 \mathrm{H}), 6.30-6.22(\mathrm{~m}$, $1 \mathrm{H}), 5.30-5.24(\mathrm{~m}, 2 \mathrm{H}), 4.65(\mathrm{~A}$ of $\mathrm{ABq}, J=11.5 \mathrm{~Hz}, 1 \mathrm{H}), 4.51(\mathrm{~A}$ of $\mathrm{ABq}, J=11.5 \mathrm{~Hz}, 1 \mathrm{H}), 4.49(\mathrm{~B}$ of $\mathrm{ABq}, J=11.5 \mathrm{~Hz}, 1 \mathrm{H}), 4.41(\mathrm{~B}$ of $\mathrm{ABq}, J=11.5 \mathrm{~Hz}, 1 \mathrm{H}), 4.44-4.38(\mathrm{~m}, 1 \mathrm{H}), 4.02-3.97(\mathrm{~m}, 1 \mathrm{H}), 3.90-3.86$ (m, 2H), 3.79 (s, 3H), 3.71 (s, 3H), 3.61 (dd, J = 2.5, 8.5 Hz, 1H), 3.04 (d, J = 3.5 Hz, -OH, 1H), 2.76 (dd, $J=10.0,4.0 \mathrm{~Hz}, 1 \mathrm{H}), 1.06(\mathrm{~s}, 9 \mathrm{H}) ;{ }^{13} \mathrm{C} N M R\left(125 \mathrm{MHz}, \mathrm{CDCl}_{3}\right) \square 159.20,159.13,142.63,142.10,135.65$, 135.20, 133.32, 133.22, 130.31, 130.27, 129.85, 129.66, 128.56, 127.71, 127.09, 127.06, 126.86, 126.77, 
117.25, 113.72, 113.66, 96.21, 78.20, 77.94, 72.87, 72.45, 71.06, 63.49, 55.19, 34.34, 26.85, 19.10; IR (thin film) $3487,2932,1513,1249 \mathrm{~cm}^{-1}$; HRMS (ES) calcd for $\mathrm{C}_{65} \mathrm{H}_{67} \mathrm{BO}_{8} \mathrm{SiNa}(\mathrm{M}+\mathrm{Na})^{+}$1037.4596, found $1037.4607 \mathrm{~m} / \mathrm{z}$.

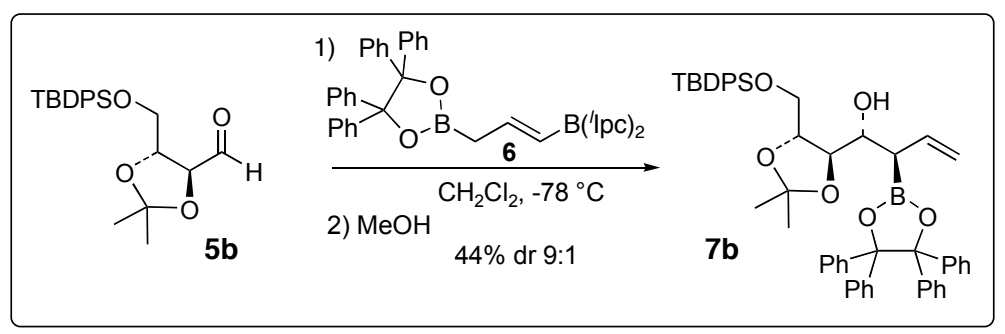

$(1 R, 2 R)-1-[(4 R, 5 R)-5$-(tert-Butyldiphenylsilanyloxymethyl)-2,2-dimethyl-[1,3]dioxolan-4-yl]-

2-(4,4,5,5-tetraphenyl-[1,3,2]dioxaborolan-2-yl)-but-3-en-1-ol (7b). 2-Allenyl-4,4,5,5-tetraphenyl-1,3,2dioxaborolane $^{3}(207 \mathrm{mg}, 0.50 \mathrm{mmol})$ and $(' \mathrm{Ipc})_{2} \mathrm{BH}^{4}(143 \mathrm{mg}, 0.50 \mathrm{mmol})$ were weighed out in a glove box. The crystalline $(' \mathrm{Ipc})_{2} \mathrm{BH}$ was ground with glass rod (to improve the rate of dissolution), then removed from the glove box and dissolved in $\mathrm{CH}_{2} \mathrm{Cl}_{2}(2 \mathrm{~mL})$. The solution was cooled to $0{ }^{\circ} \mathrm{C}$ and a solution of the above allene in $\mathrm{CH}_{2} \mathrm{Cl}_{2}(2 \mathrm{~mL})$ was added dropwise via cannula. The mixture was stirred for $2 \mathrm{~h}$ at $0{ }^{\circ} \mathrm{C}$, then was cooled to $-78{ }^{\circ} \mathrm{C}$ and a solution of aldehyde $5 \mathbf{b}^{5}$ (170 $\mathrm{mg}, 0.42 \mathrm{mmol}$ ) in $\mathrm{CH}_{2} \mathrm{Cl}_{2}$ $(1 \mathrm{~mL})$ was added dropwise. The reaction mixture was stirred for $18 \mathrm{~h}$ at $-78{ }^{\circ} \mathrm{C}$, then $\mathrm{MeOH}(2 \mathrm{~mL})$ was added and the solution was allowed to warm to ambient temperature and stirred for $4 \mathrm{~h}$. The reaction mixture was diluted with $\mathrm{CH}_{2} \mathrm{Cl}_{2}(60 \mathrm{~mL})$ and brine and the aqueous layer was extracted with $\mathrm{CH}_{2} \mathrm{Cl}_{2}(2 \mathrm{x})$. The combined organic extracts were dried over $\mathrm{Na}_{2} \mathrm{SO}_{4}$, filtered and concentrated in vacuo. The resulting oil was purified by column chromatography $\left(0.5 \% \mathrm{MeOH}\right.$ in $\left.\mathrm{CH}_{2} \mathrm{Cl}_{2}\right)$ to afford a mixture of allylboronates as a white foam (150 mg, 44\%, dr 9:1). An analytical sample of 7 a was obtained by preparative HPLC

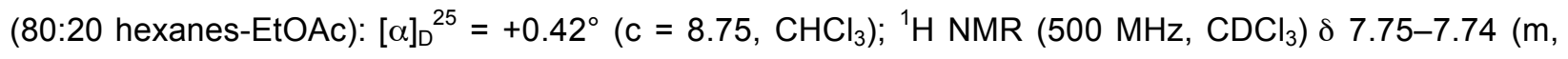
$4 \mathrm{H}), 7.45-7.41(\mathrm{~m}, 6 \mathrm{H}), 7.21-7.19(\mathrm{~m}, 8 \mathrm{H}), 7.08-7.05(\mathrm{~m}, 12 \mathrm{H}), 6.27(\mathrm{dt}, J=17.0,10.0 \mathrm{~Hz}, 1 \mathrm{H}), 5.39(\mathrm{~d}, J$ $=17.5 \mathrm{~Hz}, 1 \mathrm{H}), 5.33(\mathrm{~d}, J=10.5 \mathrm{~Hz}, 1 \mathrm{H}), 4.26(\mathrm{~m}, 1 \mathrm{H}), 4.12(\mathrm{~m}, 1 \mathrm{H}), 3.95(\mathrm{t}, J=8.0 \mathrm{~Hz}, 1 \mathrm{H}), 3.87(\mathrm{dd}, J$ $=11.0,4.5 \mathrm{~Hz}, 1 \mathrm{H}), 3.82(\mathrm{dd}, J=12.5,6.5 \mathrm{~Hz}, 1 \mathrm{H}), 3.13(\mathrm{~s}, 1 \mathrm{H}), 2.76(\mathrm{dd}, J=10.0,4.5 \mathrm{~Hz}, 1 \mathrm{H}), 1.45(\mathrm{~s}$, $3 \mathrm{H}), 1.39$ (s, 3H), $1.12(\mathrm{~s}, 9 \mathrm{H}) ;{ }^{13} \mathrm{C}$ NMR $\left(125 \mathrm{MHz}, \mathrm{CDCl}_{3}\right) \square 142.4,142.3,135.72,135.66,134.4,132.7$, 132.6, 129.9, 129.8, 128.6, 127.8, 127.7, 127.1, 126.85, 126.83, 118.1, 108.7, 96.4, 80.4, 80.1, 72.8, 65.0, 27.2, 26.9, 26.89, 26.77, 19.13; IR (thin film) 3430, 2932, 1347, $1071 \mathrm{~cm}^{-1}$; HRMS (ES) calcd for $\mathrm{C}_{52} \mathrm{H}_{55} \mathrm{BO}_{6} \mathrm{SiNa}(\mathrm{M}+\mathrm{Na})^{+} 837.3759$, found $837.3781 \mathrm{~m} / \mathrm{z}$.

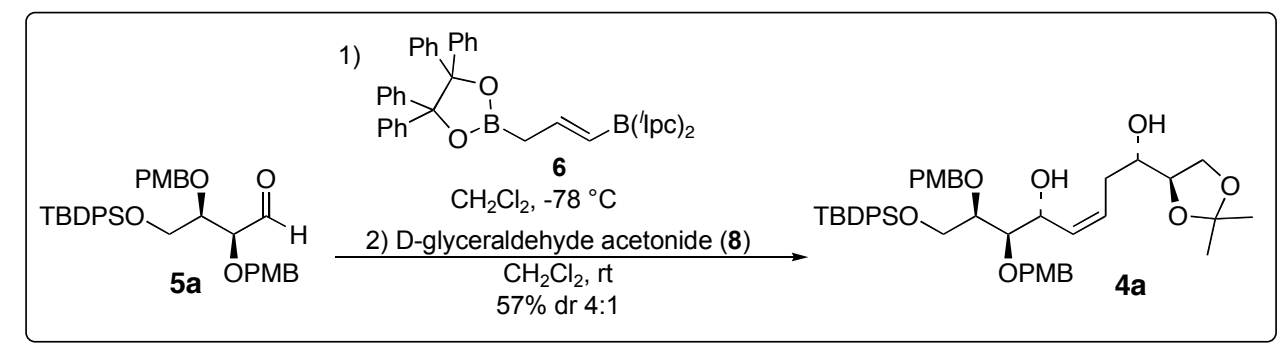




\section{(Z)-(1S,5R,6R,7R)-8-(tert-Butyldiphenylsilanyloxy)-1-((R)-2,2-dimethyl-[1,3]dioxolan-4-yl)-}

6,7-bis-(4-methoxy-benzyloxy)-oct-3-ene-1,5-diol (4a). 2-Allenyl-4,4,5,5-tetraphenyl-1,3,2dioxaborolane $^{3}(0.95 \mathrm{~g}, 2.30 \mathrm{mmol})$ and $(' \mathrm{lpc})_{2} \mathrm{BH}^{4}(0.66 \mathrm{~g}, 2.32 \mathrm{mmol})$ were weighed out in a glove box. The crystalline ('Ipc) ${ }_{2} \mathrm{BH}$ was ground with glass rod, (to improve the rate of dissolution) then removed from the glove box and dissolved in $\mathrm{CH}_{2} \mathrm{Cl}_{2}(2 \mathrm{~mL})$. The solution was cooled to $0{ }^{\circ} \mathrm{C}$ and a solution of the above allene in $\mathrm{CH}_{2} \mathrm{Cl}_{2}(2 \mathrm{~mL})$ was added dropwise via cannula. The mixture was stirred for $2 \mathrm{~h}$ at $0{ }^{\circ} \mathrm{C}$, then cooled to $-78{ }^{\circ} \mathrm{C}$ and a solution of aldehyde $5 \mathrm{a}(1.1 \mathrm{~g}, 0.82 \mathrm{mmol})$ in $\mathrm{CH}_{2} \mathrm{Cl}_{2}(0.5 \mathrm{~mL})$ was added dropwise. This mixture was stirred for $18 \mathrm{~h}$ at $-78{ }^{\circ} \mathrm{C}$, then $\mathrm{D}$-glyceraldehyde acetonide $(8)^{6}(0.60 \mathrm{~g}, 4.3$ $\mathrm{mmol}$ ) was added and the reaction was allowed to warm to ambient temperature and stir for an additional $36 \mathrm{~h}$. The reaction mixture was then cooled to $0{ }^{\circ} \mathrm{C}$, diluted with $\mathrm{CH}_{2} \mathrm{Cl}_{2}(10 \mathrm{~mL})$, and then $3 \mathrm{M} \mathrm{NaOH}$ and $\mathrm{HOOH}$ solution $(0.6 \mathrm{~mL}, 35 \%$ solution in water) were added, at which point a white precipitate formed. This mixture was stirred for $3 \mathrm{~h}$ at room temperature and then diluted with $\mathrm{CH}_{2} \mathrm{Cl}_{2}$ (50 mL), $20 \mathrm{~mL}$ of sat. $\mathrm{NaHCO}_{3}$ and $10 \mathrm{~mL}$ brine. The biphasic mixture was stirred until the precipitate had dissolved. The organic layer was separated and the aqueous washed with $\mathrm{CH}_{2} \mathrm{Cl}_{2}(2 \times 10 \mathrm{~mL})$. The organic extracts were dried over $\mathrm{Na}_{2} \mathrm{SO}_{4}$, filtered and concentrated in vacuo. The residual oil was purified by column chromatography (10\% to $66 \%$ EtOAc-hexanes) to give a mixture of 1,5 -diols (340 $\mathrm{mg}, 54 \%$ ). An analytical sample of $\mathbf{4 a}$ was obtained by HPLC purification (55:45 EtOAc-hexanes): []$_{\mathrm{D}}{ }^{25}=-17.1$ (c 1.66, $\left.\mathrm{CHCl}_{3}\right) ;{ }^{1} \mathrm{H}$ NMR (500 MHz, $\left.\mathrm{CDCl}_{3}\right) \square 7.69-7.65(\mathrm{~m}, 4 \mathrm{H}), 7.46-7.34(\mathrm{~m}, 6 \mathrm{H}), 7.16-7.09(\mathrm{~m}, 4 \mathrm{H})$, 6.84-6.76 (m, 4H), $5.63(\mathrm{dt}, J=8,11 \mathrm{~Hz}, 1 \mathrm{H}), 5.52(\mathrm{dd}, J=9,11 \mathrm{~Hz}, 1 \mathrm{H}), 4.59$ (dd, app t, $J=7.5,7.5 \mathrm{~Hz}$, $1 \mathrm{H}), 4.53(\mathrm{~A}$ of $\mathrm{ABq}, J=11.5 \mathrm{~Hz}, 1 \mathrm{H}$ ), $4.46(\mathrm{~A}$ of $\mathrm{ABq}, J=11 \mathrm{~Hz}, 1 \mathrm{H}), 4.44(\mathrm{~B}$ of $\mathrm{ABq}, J=11 \mathrm{H}, 1 \mathrm{H}), 4.37$ (B of $A B q, J=11.5 \mathrm{~Hz}, 1 \mathrm{H}), 4.11-3.96(\mathrm{~m}, 1 \mathrm{H}), 3.93-3.82(\mathrm{~m}, 4 \mathrm{H}), 3.78(\mathrm{~s}, 3 \mathrm{H}), 3.77(\mathrm{~s}, 3 \mathrm{H}), 3.71-3.67$ (m, 1H), 3.52-3.50 (m, 2H), $3.25(\mathrm{br} \mathrm{s}, 1 \mathrm{H}), 2.98(\mathrm{br} \mathrm{s}, 1 \mathrm{H}), 2.39-2.33(\mathrm{~m}, 1 \mathrm{H}), 2.20-2.12(\mathrm{~m}, 1 \mathrm{H}), 1.39$ (s, 3H), 1.34 (s, 3H), 1.06 (s, 9H); ${ }^{13} \mathrm{C}$ NMR (125 MHz, CDCl 3 ) $\square$ 159.3, 159.25, 135.6, 133.08, 133.03, 132.8, 129.97, 129.86, 129.74, 129.72, 129.68, 129.56, 128.82, 127.70, 127.68, 113.72, 113.62, 109.0, $78.74,78.72,78.1,72.9,72.2,71.1,67.1,66.3,62.5,60.3,55.15,55.12,32.05,26.8,26.6,25.3,21.0$, 19.1, 14.1; IR (thin film) 3443, 2932, 1514, $1249 \mathrm{~cm}^{-1}$; HRMS (ESI) calcd for $\mathrm{C}_{45} \mathrm{H}_{58} \mathrm{O}_{9} \mathrm{SiNa}(\mathrm{M}+\mathrm{Na})^{+}$ 793.3748 , found $793.3752 \mathrm{~m} / \mathrm{z}$.

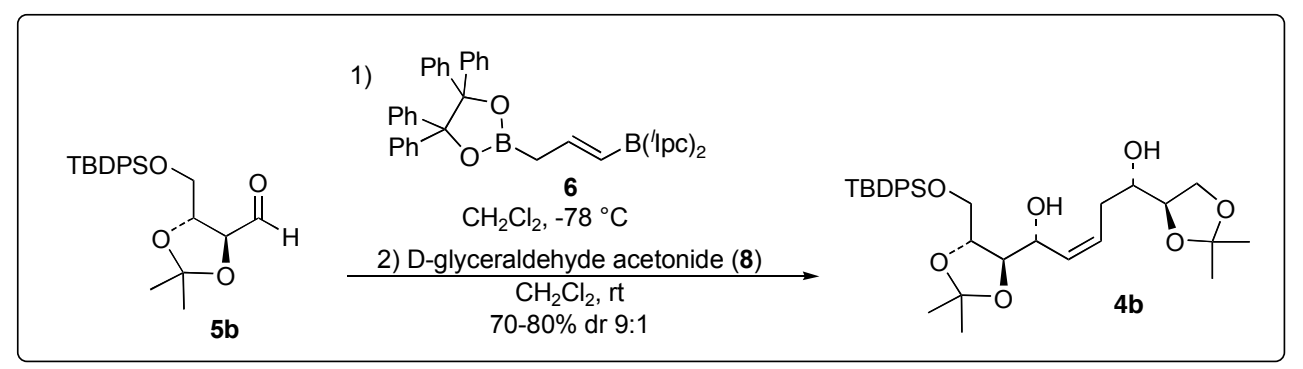

(Z)-(1R,5S)-1-[(4S,5R)-5-(tert-Butyldiphenylsilanyloxymethyl)-2,2-dimethyl-[1,3]dioxolan-4yl]-5-((R)-2,2-dimethyl-[1,3]dioxolan-4-yl)-pent-2-ene-1,5-diol (4b). 2-Allenyl-4,4,5,5-tetraphenyl-1,3,2dioxaborolane ${ }^{3}(414 \mathrm{mg}, 1.00 \mathrm{mmol})$ and $(' \mathrm{Ipc})_{2} \mathrm{BH}^{4}(286 \mathrm{mg}, 1.00 \mathrm{mmol})$ were weighed out in a glove 
box. The crystalline (' $\mathrm{Ipc})_{2} \mathrm{BH}$ was ground with glass rod (to improve the rate of dissolution) then removed from the glove box and dissolved in $\mathrm{CH}_{2} \mathrm{Cl}_{2}(2 \mathrm{~mL})$. The solution was cooled to $0{ }^{\circ} \mathrm{C}$ and a solution of the above allene in $\mathrm{CH}_{2} \mathrm{Cl}_{2}(2 \mathrm{~mL})$ was added dropwise via cannula. The mixture was stirred for $2 \mathrm{~h}$ at $0{ }^{\circ} \mathrm{C}$, then was cooled to $-78{ }^{\circ} \mathrm{C}$ and a solution of aldehyde $5 \mathbf{b}^{5}$ (326 mg, $0.800 \mathrm{mmol}$ ) in $\mathrm{CH}_{2} \mathrm{Cl}_{2}$ (0.5 mL) was added dropwise. The mixture was stirred for $18 \mathrm{~h}$ at $-78{ }^{\circ} \mathrm{C}$, then D-glyceraldehyde acetonide $(8)^{6}(221$ $\mathrm{mg}, 1.70 \mathrm{mmol}$ ) was added and the mixture was allowed to warm to ambient temperature and stir for an additional 48-64 h. Ethanolamine $(210 \mu \mathrm{L})$ was added and the solution was stirred for $2 \mathrm{~h}$, then was diluted with $\mathrm{CH}_{2} \mathrm{Cl}_{2}(30 \mathrm{~mL})$ and brine $(30 \mathrm{~mL})$. The aqueous phase was extracted with $\mathrm{CH}_{2} \mathrm{Cl}_{2}(2 \mathrm{x})$, dried over $\mathrm{Na}_{2} \mathrm{SO}_{4}$, filtered and concentrated in vacuo. The resulting oil was purified by flash column chromatography $\left(0.5 \% \mathrm{MeOH}\right.$ in $\mathrm{CH}_{2} \mathrm{Cl}_{2}$ to $30 \%$ to $50 \%$ EtOAc-hexanes) to give the title compound as a

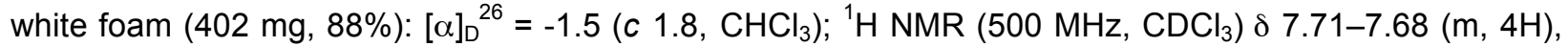
7.48-7.27 (m, 6H), 5.75-5.69 (m, 1H), $5.65(\mathrm{dd}, J=8.5 \mathrm{~Hz}, 1 \mathrm{H}), 4.52(\mathrm{t}, J=7.5 \mathrm{~Hz}, 1 \mathrm{H}), 4.09-4.04(\mathrm{~m}$, 2H), 3.96-3.84 (m, 4H), $3.76(\mathrm{dd}, J=10.5,6.5 \mathrm{~Hz}, 1 \mathrm{H}), 3.64-3.60(\mathrm{~m}, 1 \mathrm{H}), 3.26(\mathrm{br} \mathrm{s}, 1 \mathrm{H}), 2.91(\mathrm{~d}, J=$ $3.0 \mathrm{~Hz}, 1 \mathrm{H}$ ), 2.51 (dddd, $J=13.0,5.5,3.5,2.5 \mathrm{~Hz}, 1 \mathrm{H}$ ), 2.35 (dt, $J=14.0,9.0 \mathrm{~Hz}, 1 \mathrm{H}$ ), 1.43 (s, 3H), 1.42 (s, 3H), 1.36 (s, 6H), 1.08 (s, 9H); ${ }^{13} \mathrm{C}$ NMR (125 MHz, $\left.\mathrm{CDCl}_{3}\right) \square 135.61,135.60,132.4,132.4,132.1$, 130.0, 129.9, 129.7, 127.83, 127.81, 109.18, 109.16, 81.3, 79.5, 78.5, 71.2, 68.1, 66.5, 64.5, 32.5, 26.8, 26.7, 26.7, 26.6, 25.3, 19.1; IR (thin film) 3453, 2932, $1069 \mathrm{~cm}^{-1}$; Anal calcd for $\mathrm{C}_{32} \mathrm{H}_{46} \mathrm{O}_{7} \mathrm{Si}$ : C, 67.34; $\mathrm{H}$, 8.12. Found: C, $66.95 ; \mathrm{H}, 8.42$.

Stereochemistry of $\mathbf{4 b w a s}$ assignment via modified Mosher ester analysis (values in $\mathrm{Hz}, \mathrm{CD}=\square \mathrm{s}-$ ५R):
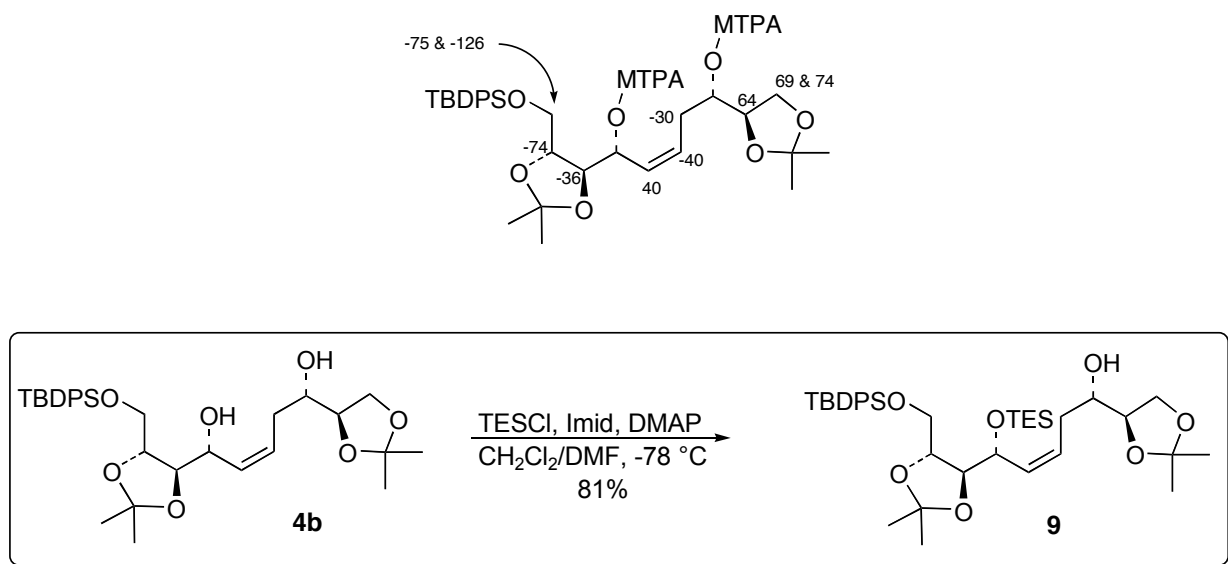

(Z)-(1S,5R)-5-[(4R,5R)-5-(tert-Butyldiphenylsilanyloxymethyl)-2,2-dimethyl-[1,3]dioxolan-4yl]-1-((R)-2,2-dimethyl-[1,3]dioxolan-4-yl)-5-triethylsilanyloxypent-3-en-1-ol (9). To a $-78{ }^{\circ} \mathrm{C}$ solution of imidazole (628 mg, $9.24 \mathrm{mmol}$ ), DMAP (6.0 mg, $42 \mu \mathrm{mol})$, and 1,5-diol $4 \mathbf{b}(4.8 \mathrm{~g}, 8.4 \mathrm{mmol})$ in $\mathrm{CH}_{2} \mathrm{Cl}_{2}$ $(61 \mathrm{~mL})$ and DMF $(61 \mathrm{~mL})$ was added a solution of chlorotriethylsilane $(1.48 \mathrm{~mL}, 8.8 \mathrm{mmol})$ in $\mathrm{CH}_{2} \mathrm{Cl}_{2}(15$ $\mathrm{mL}$ ) dropwise via cannula. The reaction mixture was stirred for $3 \mathrm{~h}$ at $-78{ }^{\circ} \mathrm{C}$, then allowed to warm to room temperature, and diluted with $50 \%$ EtOAc-hexane and sat. $\mathrm{NH}_{4} \mathrm{Cl}$. The biphasic mixture was separated and the organic phase was washed with sat. $\mathrm{NaHCO}_{3}$, and brine, then dried over $\mathrm{Na}_{2} \mathrm{SO}_{4}$, filtered, and concentrated in vacuo. The resulting oil was purified by flash column chromatography $(10 \%$ 


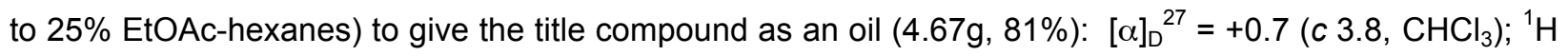
NMR (400 MHz, $\left.\mathrm{CDCl}_{3}\right) \square 7.75-7.73(\mathrm{~m}, 4 \mathrm{H}), 7.43-7.37(\mathrm{~m}, 6 \mathrm{H}), 5.61-5.57(\mathrm{~m}, 2 \mathrm{H}), 4.45(\mathrm{t}, \mathrm{J}=8.0 \mathrm{~Hz}$, 1H), 4.15-4.09 (m, 2H), 4.01-3.86 (m, 4H), 3.76 (dd, J = 11.2, $4.0 \mathrm{~Hz}, 1 \mathrm{H}), 3.56-3.51(\mathrm{~m}, 1 \mathrm{H}), 3.24(\mathrm{~d}, J$ $=4.0 \mathrm{~Hz}, 1 \mathrm{H}), 2.55(\mathrm{~d}, J=14.0 \mathrm{~Hz}, 1 \mathrm{H}), 2.30(\mathrm{dt}, J=13.6,10.0 \mathrm{~Hz}, 1 \mathrm{H}), 1.46(\mathrm{~s}, 3 \mathrm{H}), 1.44(\mathrm{~s}, 3 \mathrm{H}), 1.43$ (s, 3H), $1.38(\mathrm{~s}, 3 \mathrm{H}), 1.07$ (s, 9H), $0.91(\mathrm{t}, J=8 \mathrm{~Hz}, 9 \mathrm{H}), 0.55$ (q, J = 8.0 Hz, 6H); ${ }^{13} \mathrm{C}$ NMR (100 MHz, $\left.\mathrm{CDCl}_{3}\right) \square 135.7,135.6,134.7,133.5,133.4,129.5,128.0,127.6,127.5,109.3,109.2,81.5,79.2,77.9$, 71.8, 70.6, 67.2, 64.5, 33.2, 27.0, 26.9, 26.7, 26.7, 25.3, 19.3, 6.7, 4.9; IR (thin film) 3486, 2877, $1073 \mathrm{~cm}^{-}$ ${ }^{1}$; HRMS (ESI) calcd for calcd for $\mathrm{C}_{38} \mathrm{H}_{60} \mathrm{O}_{7} \mathrm{Si}_{2} \mathrm{Na} 707.3775 \mathrm{~m} / \mathrm{z}(\mathrm{M}+\mathrm{Na})^{+}$; observed $707.3779 \mathrm{~m} / \mathrm{z}$.

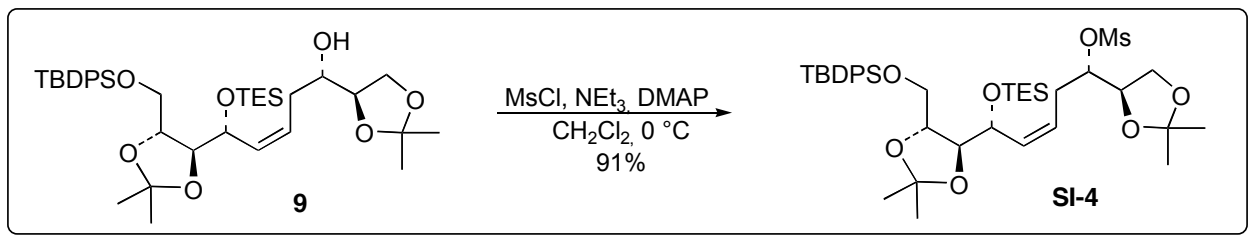

Methanesulfonic acid (Z)-(1S,5R)-5-[(4R,5R)-5-(tert-butyldiphenylsilanyloxymethyl)-2,2dimethyl-[1,3]dioxolan-4-yl]-1-((R)-2,2-dimethyl-[1,3]dioxolan-4-yl)-5-triethylsilanyloxypent-3-enyl ester (SI-4). To a $0{ }^{\circ} \mathrm{C}$ solution of alcohol $9(7.19 \mathrm{~g}, 10.5 \mathrm{mmol})$, triethylamine $(7.37 \mathrm{~mL}, 52.5 \mathrm{mmol})$, and DMAP (128 mg, $1.1 \mathrm{mmol})$ in $\mathrm{CH}_{2} \mathrm{Cl}_{2}(104 \mathrm{~mL})$ was added methanesulfonyl chloride $(8.13 \mathrm{~mL}, 105 \mathrm{mmol})$. The reaction mixture was stirred $30 \mathrm{~min}$, then diluted with $\mathrm{CH}_{2} \mathrm{Cl}_{2}$ and aq. $\mathrm{NH}_{4} \mathrm{Cl}$. The organic layer was washed with $1 \mathrm{M} \mathrm{NaOH}$, sat. $\mathrm{NaHCO}_{3}$, and brine; then dried over $\mathrm{Na}_{2} \mathrm{SO}_{4}$, filtered, and concentrated in vacuo. The residue was purified by flash column chromatography (30\% EtOAc-hexanes) to give the title

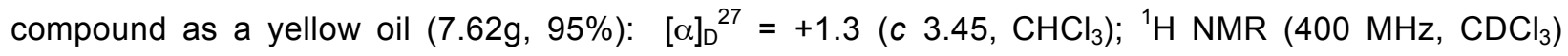
77.72-7.69 (m, 4H), 7.41-7.32 (m, 6H), 5.57-5.46 (m, 2H), $4.78(\mathrm{q}, J=5.6 \mathrm{~Hz}, 1 \mathrm{H}), 4.45$ (dd, J = 7.6, 6.4 $\mathrm{Hz}, 1 \mathrm{H}), 4.15-4.10(\mathrm{~m}, 2 \mathrm{H}), 4.04(\mathrm{t}, J=6.4 \mathrm{~Hz}, 1 \mathrm{H}), 3.93-3.88(\mathrm{~m}, 3 \mathrm{H}), 3.71(\mathrm{dd}, J=11.2,4.4 \mathrm{~Hz}, 1 \mathrm{H})$, $3.01(\mathrm{~s}, 3 \mathrm{H}), 2.65-2.52(\mathrm{~m}, 2 \mathrm{H}), 1.42(\mathrm{~s}, 3 \mathrm{H}), 1.40(\mathrm{~s}, 3 \mathrm{H}), 1.39(\mathrm{~s}, 3 \mathrm{H}), 1.31(\mathrm{~s}, 3 \mathrm{H}), 1.04(\mathrm{~s}, 9 \mathrm{H}), 0.90(\mathrm{t}$, $8.0 \mathrm{~Hz}, 9 \mathrm{H}), 0.53$ (q, $8.0 \mathrm{~Hz}, 6 \mathrm{H}) ;{ }^{13} \mathrm{C}$ NMR $\left(100 \mathrm{MHz}, \mathrm{CDCl}_{3}\right) \square 135.7,135.6,134.5,133.4,129.6,127.61$, 127.59, 124.6, 109.7, 109.2, 80.6, 80.0, 79.1, 75.9, 70.0, 65.3, 64.8, 38.7, 30.6, 27.18, 27.16, 26.8, 26.3, 25.2, 19.3; IR (thin film) 2935, $1081 \mathrm{~cm}^{-1}$; HRMS (ESI) calcd for $\mathrm{C}_{39} \mathrm{H}_{62} \mathrm{O}_{9} \mathrm{SSi}_{2} \mathrm{Na} 785.3551 \mathrm{~m} / \mathrm{z}(\mathrm{M}+\mathrm{Na})^{+}$; observed $785.3567 \mathrm{~m} / \mathrm{z}$.

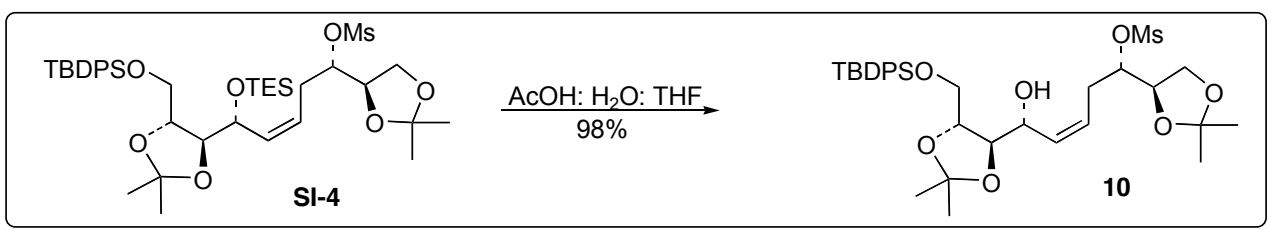

Methanesulfonic acid (Z)-(1S,5R)-5-[(4S,5R)-5-(tert-butyldiphenylsilanyloxymethyl)-2,2dimethyl-[1,3]dioxolan-4-yl]-1-((R)-2,2-dimethyl-[1,3]dioxolan-4-yl)-5-hydroxy-pent-3-enyl ester (10). A mixture of silyl ether SI-4 $(9.18 \mathrm{~g}, 12.0 \mathrm{mmol})$, acetic acid $(57 \mathrm{~mL})$, water $(19 \mathrm{~mL})$, and THF $(19 \mathrm{~mL})$ was stirred at ambient temperature until the reaction was judged complete by TLC analysis $(1.5 \mathrm{~h})$. The 
reaction mixture was diluted with EtOAc (1L) and neutralized with $\mathrm{NaOH}(500 \mathrm{~mL}, 3 \mathrm{M})$. The biphasic mixture was separated and the organic layer was washed with $\mathrm{NaOH}(3 \mathrm{M})$, sat. $\mathrm{NaHCO}_{3}$, and brine; then dried over $\mathrm{Na}_{2} \mathrm{SO}_{4}$, filtered and concentrated in vacuo. The resulting oil was purified by flash column chromatography (45\% EtOAc-hexane) to give the title compound as a white foam $(7.65 \mathrm{~g}, 98 \%)$ : [0] ${ }_{\mathrm{D}}{ }^{27}=$ 0.93 (c 3.1, $\left.\mathrm{CHCl}_{3}\right) ;{ }^{1} \mathrm{H}$ NMR $\left(500 \mathrm{MHz}, \mathrm{CDCl}_{3}\right) \square 7.71-7.68(\mathrm{~m}, 4 \mathrm{H}), 7.48-7.40(\mathrm{~m}, 6 \mathrm{H}), 5.72-5.63(\mathrm{~m}$, $2 \mathrm{H}), 4.79(\mathrm{q}, J=6.0 \mathrm{~Hz}, 1 \mathrm{H}), 4.53-4.49(\mathrm{~m}, 1 \mathrm{H}), 4.20(\mathrm{q}, J=6.0 \mathrm{~Hz}, 1 \mathrm{H}), 4.09-4.04(\mathrm{~m}, 2 \mathrm{H}), 3.98-3.93$ $(\mathrm{m}, 2 \mathrm{H}), 3.82(\mathrm{dd}, J=11.0,4.0 \mathrm{~Hz}, 1 \mathrm{H}), 3.77(\mathrm{dd}, J=11.0,5.5 \mathrm{~Hz}, 1 \mathrm{H}), 3.06(\mathrm{~s}, 3 \mathrm{H}), 2.93(\mathrm{~d}, J=2.5 \mathrm{~Hz}$, $1 \mathrm{H}), 2.72-2.60(\mathrm{~m}, 2 \mathrm{H}), 1.43(\mathrm{~s}, 3 \mathrm{H}), 1.43(\mathrm{~s}, 3 \mathrm{H}), 1.39(\mathrm{~s}, 3 \mathrm{H}), 1.35(\mathrm{~s}, 3 \mathrm{H}), 1.08(\mathrm{~s}, 9 \mathrm{H}) ;{ }^{13} \mathrm{C}$ NMR $(100$ $\left.\mathrm{MHz}, \mathrm{CDCl}_{3}\right) \square$ 135.6, 132.6, 132.2, 129.92, 129.88, 127.8, 127.0, 109.8, 109.1, 80.9, 80.4, 78.9, 75.5, $67.9,65.4,64.7,38.7,30.2,26.93,26.90,26.8,26.4,25.1,19.1$; IR (thin film) $3467,2933,1175 \mathrm{~cm}^{-1}$; HRMS (ESI) calcd for $\mathrm{C}_{33} \mathrm{H}_{48} \mathrm{O}_{9} S S i N a 671.2686 \mathrm{~m} / \mathrm{z}(\mathrm{M}+\mathrm{Na})^{+}$; observed $671.2692 \mathrm{~m} / \mathrm{z}$.

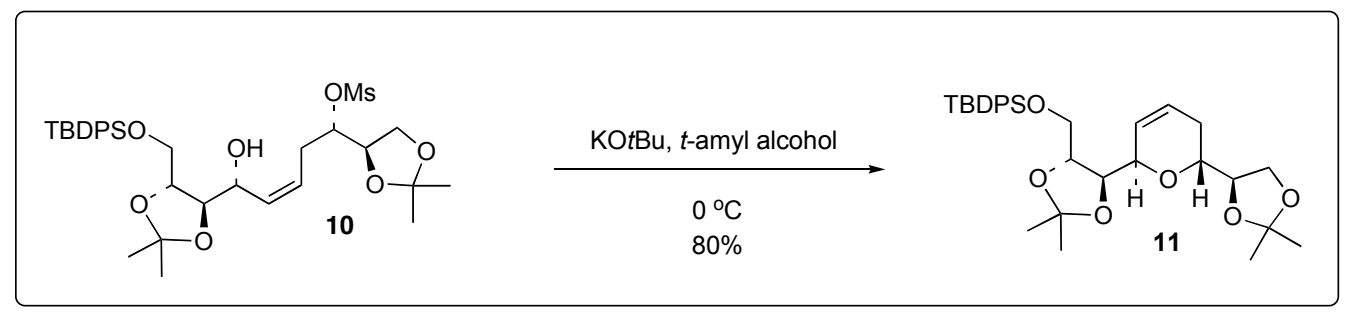

tert-Butyl-\{(4R,5S)-5-[(2R,6R)-6-((R)-2,2-dimethyl-[1,3]dioxolan-4-yl)-5,6-dihydro-2H-pyran-

2-yl]-2,2-dimethyl-[1,3]dioxolan-4-ylmethoxy\}-diphenylsilane (11). To a suspension of $\mathrm{KOtBu}(1.40 \mathrm{~g}$, $12.5 \mathrm{mmol})$ in $t$-amyl alcohol $(60 \mathrm{~mL})$ at $0{ }^{\circ} \mathrm{C}$ was added dropwise a solution of homoallylic mesylate 10 $(5.4 \mathrm{~g}, 8.24 \mathrm{mmol})$ in $t$-amyl alcohol $(160 \mathrm{~mL})$. The mixture was stirred for $24 \mathrm{~h}$ at $4{ }^{\circ} \mathrm{C}$, then was diluted with EtOAc and washed with sat. $\mathrm{NaHCO}_{3}$. The aqueous phase was then extracted with EtOAc (3x), and the combine organics were washed with brine, dried over $\mathrm{Na}_{2} \mathrm{SO}_{4}$, filtered and concentrated in vacuo. The resulting oil (an 85 : 15 mixture of 11 and diene 12) was purified by flash column chromatography ( $10 \%$ to $25 \%$ EtOAc-hexane) to give the title compound as a clear oil $(3.72 \mathrm{~g}, 80 \%): \quad[\square]_{\mathrm{D}}^{27}=+6.2(c 4.2$, $\left.\mathrm{CHCl}_{3}\right) ;{ }^{1} \mathrm{H}$ NMR $\left(400 \mathrm{MHz}, \mathrm{CDCl}_{3}\right) \square 7.58-7.54(\mathrm{~m}, 4 \mathrm{H}), 7.27-7.19(\mathrm{~m}, 6 \mathrm{H}), 5.79(\mathrm{dt}, \mathrm{J}=10.4,2.4 \mathrm{~Hz}$, $1 \mathrm{H}), 5.74(\mathrm{~d}, J=11.2 \mathrm{~Hz}, 1 \mathrm{H}), 4.00(\mathrm{bs}, 1 \mathrm{H}), 3.96-3.91(\mathrm{~m}, 2 \mathrm{H}), 3.86(\mathrm{q}, J=6.4 \mathrm{~Hz}, 1 \mathrm{H}), 3.81(\mathrm{dd}, J=$ $10.8,3.2 \mathrm{~Hz}, 1 \mathrm{H}), 3.70(\mathrm{dd}, J=8.4,6.7 \mathrm{~Hz}, 1 \mathrm{H}), 3.66-3.54(\mathrm{~m}, 3 \mathrm{H}), 1.83-1.78(\mathrm{~m}, 2 \mathrm{H}), 1.26(\mathrm{~s}, 3 \mathrm{H}), 1.23$ (s, 3H), $1.16(\mathrm{~s}, 3 \mathrm{H}), 1.14(\mathrm{~s}, 3 \mathrm{H}), 0.91(\mathrm{~s}, 9 \mathrm{H}) ;{ }^{13} \mathrm{C}$ NMR (100 MHz, $\left.\mathrm{CDCl}_{3}\right) \square$ 139.4, 135.0, 133.7, 133.6, 129.9, 129.8, 127.9, 127.8, 126.7, 124.9, 109.7, 109.5, 81.1, 78.4, 77.1, 74.3, 69.9, 65.4, 64.6, 27.6, 27.5, 27.1, 26.8, 26.5, 25.33, 25.28, 19.5; IR (thin film) 2931, $1073 \mathrm{~cm}^{-1}$; HRMS (ESI) calcd for $\mathrm{C}_{32} \mathrm{H}_{44} \mathrm{O}_{6} \mathrm{SiNa}$ $575.2805 \mathrm{~m} / \mathrm{z}(\mathrm{M}+\mathrm{Na})^{+}$; observed $575.2809 \mathrm{~m} / \mathrm{z}$. 


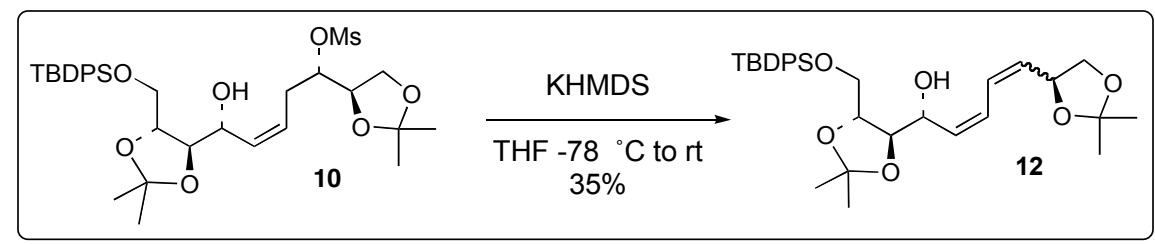

(2Z,4Z)-(R)-1-[(4S,5R)-5-(tert-Butyl-diphenyl-silanyloxymethyl)-2,2-dimethyl-[1,3]dioxolan-4-

yl]-5-((S)-2,2-dimethyl-[1,3]dioxolan-4-yl)-penta-2,4-dien-1-ol (12). To a $-78{ }^{\circ} \mathrm{C}$ solution of hydroxy mesylate $10(57 \mathrm{mg}, 87 \mu \mathrm{mol})$ in THF $(0.5 \mathrm{~mL})$ was added KHMDS (211uL, $0.105 \mathrm{mmol}, 0.5 \mathrm{M}$ in toluene). The reaction mixture was stirred $0.5 \mathrm{~h}$ at $-78{ }^{\circ} \mathrm{C}$, allowed to warm to ambient temperature, diluted with sat. $\mathrm{NH}_{4} \mathrm{Cl}(1 \mathrm{~mL})$ and EtOAc. The biphasic mixture was separated and the aqueous phase was extracted with EtOAc (2x). The combined organic layers were washed with brine, dried over $\mathrm{Na}_{2} \mathrm{SO}_{4}$, filtered and concentrated in vacuo. The resulting oil was purified by flash column chromatography $(10 \%$

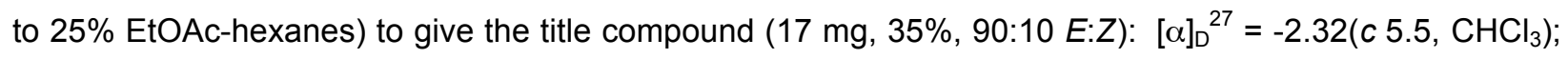
${ }^{1} \mathrm{H}$ NMR $\left(500 \mathrm{MHz}, \mathrm{CDCl}_{3}\right) \square 7.62-7.57(\mathrm{~m}, 4 \mathrm{H}), 7.39-7.18(\mathrm{~m}, 6 \mathrm{H}), 6.54(\mathrm{dd}, J=15.0,11.5 \mathrm{~Hz}, 1 \mathrm{H}), 6.06$ (t, $J=11.5 \mathrm{~Hz}, 1 \mathrm{H}), 5.63(\mathrm{dd}, J=15.0,7.5 \mathrm{~Hz}, 1 \mathrm{H}), 5.35(\mathrm{dd}, J=11.0,8.5 \mathrm{~Hz}, 1 \mathrm{H}), 4.69-4.66(\mathrm{~m}, 1 \mathrm{H})$, $4.41(\mathrm{q}, J=7.5 \mathrm{~Hz}, 1 \mathrm{H}), 4.03-3.93(\mathrm{~m}, 3 \mathrm{H}), 3.71$ (dd, $J=11.0,4.5 \mathrm{~Hz}, 1 \mathrm{H}), 3.67$ (dd, $J=11.0,3.5 \mathrm{~Hz}$, 1H), $3.48(\mathrm{t}, J=8.0 \mathrm{~Hz}, 1 \mathrm{H}), 2.47(\mathrm{~d}, J=3 \mathrm{~Hz}, 1 \mathrm{H}), 1.35$ (s, 6H), $1.34(\mathrm{~s}, 3 \mathrm{H}), 1.31(\mathrm{~s}, 3 \mathrm{H}), 0.98(\mathrm{~s}, 9 \mathrm{H})$; ${ }^{13} \mathrm{C}$ NMR $\left(125 \mathrm{MHz}, \mathrm{CDCl}_{3}\right) \square$ 135.62, 135.60, 133.0, 132.9, 132.8, 131.0, 129.9, 129.8, 129.6, 128.2, $127.8,127.7,109.5,109.2,80.5,77.9,76.6,69.3,68.0,64.5,27.0,26.8,26.7,25.9,19.2$; IR (thin film) $3450,2932,1061 \mathrm{~cm}^{-1}$. Anal calcd for $\mathrm{C}_{32} \mathrm{H}_{44} \mathrm{O}_{6}: \mathrm{C}, 69.53 ; \mathrm{H}, 8.02$. Found: $\mathrm{C}, 69.37 ; \mathrm{H}, 8.35$.

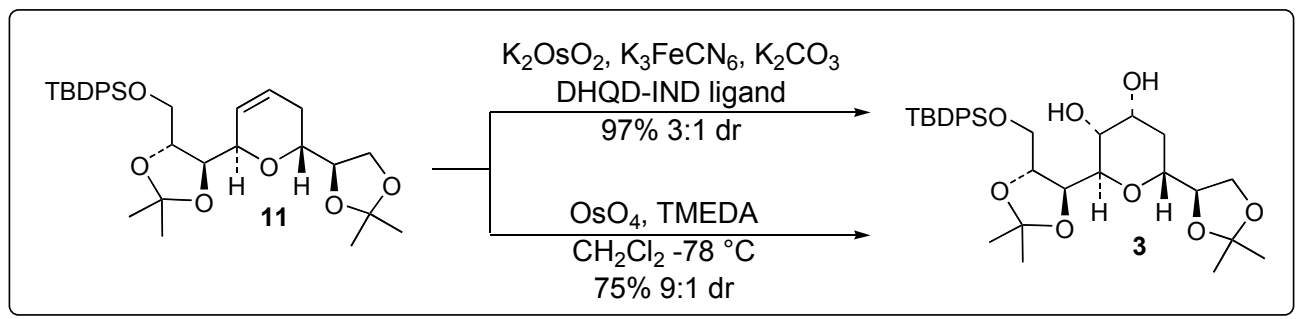

$(2 R, 3 R, 4 R, 6 R)-2-[(4 R, 5 R)-5-(t e r t-B u t y l d i p h e n y l s i l a n y l o x y m e t h y l)-2,2-d i m e t h y l-[1,3]$ dioxolan-

\section{4-yl]-6-((R)-2,2-dimethyl-[1,3]dioxolan-4-yl)-tetrahydropyran-3,4-diol (3).}

Sharpless Asymmetric Dihydroxylation: To a $0{ }^{\circ} \mathrm{C}$ solution of dihydropyran $11(1.52 \mathrm{~g}, 2.75 \mathrm{mmol})$ in $t$ - $\mathrm{BuOH}(68 \mathrm{~mL})$ and water $(68 \mathrm{~mL})$ was added methanesulfonamide $(510 \mathrm{mg}, 5.4 \mathrm{mmol})$ and $28 \mathrm{~g}$ of a stock mixture composed of $\mathrm{K}_{3} \mathrm{FeCN}_{6}(31.6 \mathrm{~g}), \mathrm{K}_{2} \mathrm{CO}_{3}(13.4 \mathrm{~g}), \mathrm{K}_{2} \mathrm{OsO}_{2}(\mathrm{OH})_{4}(46 \mathrm{mg})$, DHQD-Ind ligand ${ }^{7}$ $(284 \mathrm{mg})$. The reaction mixture was stirred for $24 \mathrm{~h}$ at $4{ }^{\circ} \mathrm{C}$, then $\mathrm{Na}_{2} \mathrm{~S}_{2} \mathrm{O}_{3}(30 \mathrm{~g})$ was added and the resulting solution was stirred for $2 \mathrm{~h}$, after which it was diluted with EtOAc and water. The biphasic mixture was separated and the aqueous phase was extracted with EtOAc (3x). The combined organic layers were washed with $1 \mathrm{~N} \mathrm{KOH}$, sat. $\mathrm{NaHCO}_{3}$, and brine, dried over $\mathrm{Na}_{2} \mathrm{SO}_{4}$, filtered, and concentrated in vacuo. The resulting foam (1.56 g, 97\%, 3:1 mixture of diastereomers) was purified by flash column chromatography (60\% EtOAc: hexane) to give the major diasteromer $3(1.07 \mathrm{~g}, 66 \%)$. 
Stoichiometric Dihydroxylation: To a solution of dihydropyran 11 (30 mg, $54 \mu \mathrm{mol})$ in $\mathrm{CH}_{2} \mathrm{Cl}_{2}(5$ $\mathrm{mL})$ at $-78{ }^{\circ} \mathrm{C}$ was added TMEDA $(9 \mu \mathrm{L}, 60 \mu \mathrm{mol})$ and $\mathrm{OsO}_{4}(285 \mu \mathrm{L}, 57 \mu \mathrm{mol}, 0.2 \mathrm{M}$ in toluene). The mixture was stirred for $1 \mathrm{~h}$, then the solvent was removed under a stream of $\mathrm{N}_{2}$. The residue was diluted with THF $(5 \mathrm{~mL})$, acetone $(2.5 \mathrm{~mL})$, water $(2.5 \mathrm{~mL})$, and treated with $\mathrm{Na}_{2} \mathrm{~S}_{2} \mathrm{O}_{3}(450 \mathrm{mg})$. This mixture was heated to reflux for $3 \mathrm{~h}$, diluted with EtOAc and water, and the aqueous phase was extracted with EtOAc (2x). The combined organic extracts were concentrated in vacuo to give an oil, which was resubjected to treatment with THF/acetone/water/ $/ \mathrm{Na}_{2} \mathrm{~S}_{2} \mathrm{O}_{3}$ at reflux for $4 \mathrm{~d}$ to allow for complete hydrolysis of the osmate ester. The solution was diluted with EtOAc and water and the aqueous phase extracted with EtOAc (2x). The combined organic extracts were washed with brine, dried over $\mathrm{Na}_{2} \mathrm{SO}_{4}$, filtered and concentrated in vacuo. The oil was passed though a $\mathrm{SiO}_{2}$ plug (eluted with $60 \%$ EtOAc-hexane) to give a $9: 1$ mixture of diastereomers containing the title compound as the major isomer (24 mg, 75\%). Data for major diastereomer 3: $[\square]_{D}{ }^{27}=+0.56\left(c 3.3, \mathrm{CHCl}_{3}\right) ;{ }^{1} \mathrm{H}$ NMR $\left(500 \mathrm{MHz}, \mathrm{CDCl}_{3}\right) \square 7.74-7.70(\mathrm{~m}, 4 \mathrm{H}), 7.43-7.27$ $(\mathrm{m}, 6 \mathrm{H}), 4.50(\mathrm{q}, J=7.0 \mathrm{~Hz}, 1 \mathrm{H}), 4.22-4.15(\mathrm{~m}, 2 \mathrm{H}), 4.11-4.08(\mathrm{~m}, 1 \mathrm{H}), 4.01-3.96(\mathrm{~m}, 2 \mathrm{H}), 3.86(\mathrm{t}, J=$ $7.0 \mathrm{~Hz}, 1 \mathrm{H}), 3.82(\mathrm{dd}, J=11.5,4.0 \mathrm{~Hz}, 1 \mathrm{H}), 3.76(\mathrm{dt}, J=6.5,3.0 \mathrm{~Hz}, 1 \mathrm{H}), 3.71-3.68(\mathrm{~m}, 1 \mathrm{H}), 3.65(\mathrm{dd}, J$ $=8.0,6.0 \mathrm{~Hz}, 1 \mathrm{H}), 3.23(\mathrm{~d}, J=3.5 \mathrm{~Hz}, 1 \mathrm{H}), 2.80(\mathrm{~d}, J=4.5 \mathrm{~Hz}, 1 \mathrm{H}), 1.91(\mathrm{dt}, J=14.5,5.0 \mathrm{~Hz}, 1 \mathrm{H}), 1.73$ (ddd, $J=14.5,6.0,5.0 \mathrm{~Hz}, 1 \mathrm{H}), 1.47(\mathrm{~s}, 3 \mathrm{H}), 1.45(\mathrm{~s}, 3 \mathrm{H}), 1.33(\mathrm{~s}, 3 \mathrm{H}), 1.32(\mathrm{~s}, 3 \mathrm{H}), 1.07(\mathrm{~s}, 9 \mathrm{H}) ;{ }^{13} \mathrm{C}$ NMR $\left(125 \mathrm{MHz}, \mathrm{CDCl}_{3}\right) \square$ 136.0, 135.9, 133.7, 133.6, 129.9, 129.8, 127.8, 110.2, 109.8, 81.9, 77.2, 75.9, $73.4,72.1,70.3,66.3,65.7,63.8,30.7,27.4,27.3,27.1,27.0,25.7,19.5$; IR (thin film) 3435, 2932, 1113 $\mathrm{cm}^{-1}$; HRMS (ESI) calcd for $\mathrm{C}_{32} \mathrm{H}_{46} \mathrm{O}_{8} \mathrm{SiNa} 609.2860 \mathrm{~m} / \mathrm{z}(\mathrm{M}+\mathrm{Na})^{+}$; observed $609.2861 \mathrm{~m} / \mathrm{z}$.

Data for minor diol diastereomer: $[\square]_{D}^{27}=+0.52$ (c 5.6, $\left.\mathrm{CHCl}_{3}\right) ;{ }^{1} \mathrm{H}$ NMR $\left(500 \mathrm{MHz}, \mathrm{CDCl}_{3}\right)$ प7.64-7.62 (m, 4H), 7.26-7.19 (m, 6H), $4.32(\mathrm{t}, J=7.5 \mathrm{~Hz}, 1 \mathrm{H}), 4.12-4.09(\mathrm{~m}, 1 \mathrm{H}), 4.03(\mathrm{q}, J=7 \mathrm{~Hz}$, 1H), $3.98(\mathrm{br} \mathrm{s}, 1 \mathrm{H}), 3.92(\mathrm{br} \mathrm{s}, 1 \mathrm{H}), 3.87-3.81(\mathrm{~m}, 3 \mathrm{H}), 3.77-3.74(\mathrm{~m}, 1 \mathrm{H}), 3.72(\mathrm{dd}, J=11.0,4.5 \mathrm{~Hz}$, 1H), 3.61 (dd, $J=8.0,7.0 \mathrm{~Hz}, 1 \mathrm{H}$ ), 2.99 (br d, $J=4.5 \mathrm{~Hz}, 1 \mathrm{H}$ ), 2.35 (br s, 1H), 1.94 (ddd, $J=13.5,10.0$, $6.0 \mathrm{~Hz}, 1 \mathrm{H}), 1.61(\mathrm{dt}, J=13.5,4.5 \mathrm{~Hz}, 1 \mathrm{H}), 1.36(\mathrm{~s}, 3 \mathrm{H}), 1.35(\mathrm{~s}, 3 \mathrm{H}), 1.25(\mathrm{~s}, 3 \mathrm{H}), 1.21(\mathrm{~s}, 3 \mathrm{H}), 0.99(\mathrm{~s}$, 9H); ${ }^{13} \mathrm{C}$ NMR $\left(125 \mathrm{MHz}, \mathrm{CDCl}_{3}\right) \square 135.8,135.7,129.7,129.6,127.6,109.8,109.7,80.9,77.03,75.9$, $74.8,70.7,68.5,66.2,65.9,64.1,30.7,27.3,27.2,26.9,26.6,25.1,19.3$; IR (thin film) 3413, 2932, 1112 $\mathrm{cm}^{-1}$.

Stereochemical assignment of major diastereomer 3 was based on coupling constant and ${ }^{1} \mathrm{H}$ nOe analysis:

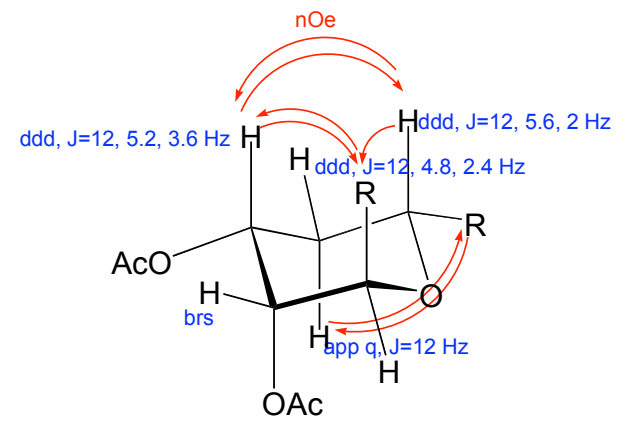




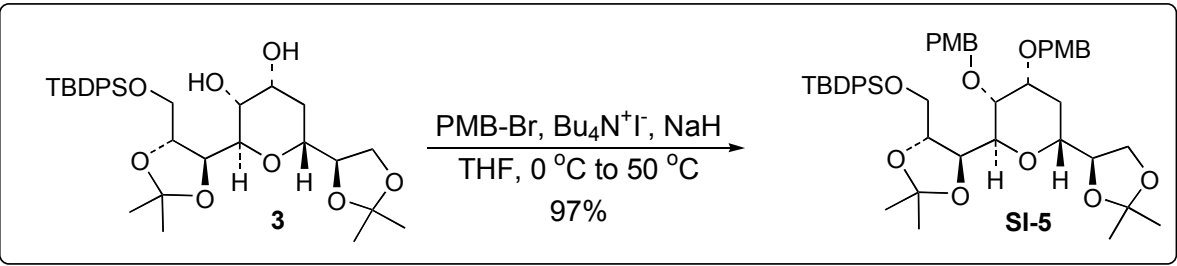

tert-Butyl-\{(4R,5R)-5-[(2R,3R,4R,6R)-6-((R)-2,2-dimethyl-[1,3]dioxolan-4-yl)-3,4-bis-(4methoxy-benzyloxy)-tetrahydropyran-2-yl]-2,2-dimethyl-[1,3]dioxolan-4-ylmethoxy\}-diphenylsilane

(SI-5). To a $0{ }^{\circ} \mathrm{C}$ suspension of $\mathrm{NaH}(48 \mathrm{mg}, 1.2 \mathrm{mmol}, 60 \%$ in mineral oil) in THF (2 mL) was added dropwise a solution of diol $3(320 \mathrm{mg}, 0.54 \mathrm{mmol}), \mathrm{PMB}-\mathrm{Br}(271 \mathrm{mg}, 1.35 \mathrm{mmol})$, and tetrabutylammonium iodide $(10 \mathrm{mg}, 27 \mu \mathrm{mol})$ as a solution in THF $(3.5 \mathrm{~mL})$. The reaction mixture was warmed to ambient temperature, the flask was equipped with a reflux condenser, and the solution was heated to reflux. Upon completion of the reaction as judged by TLC analysis (5h), the reaction mixture was cooled to ambient temperature and diluted with sat. $\mathrm{NaHCO}_{3}$ and $\mathrm{EtOAc}$. The organic phase was washed with $\mathrm{NaHCO}_{3}$, and brine, dried over $\mathrm{Na}_{2} \mathrm{SO}_{4}$, filtered and concentrated in vacuo. The resulting oil was purified by column chromatography ( $10 \%$ to $25 \%$ EtOAc-hexane) to give the title compound as a white foam (435 mg, 97\%): [ []$_{\mathrm{D}}{ }^{27}=+2.0\left(\mathrm{c} \mathrm{4.9}, \mathrm{CHCl}_{3}\right) ;{ }^{1} \mathrm{H}$ NMR $\left(500 \mathrm{MHz}, \mathrm{CDCl}_{3}\right) \square 7.72(\mathrm{~d}, \mathrm{~J}=8.0 \mathrm{~Hz}$, $2 \mathrm{H}), 7.68(\mathrm{~d}, J=8.0 \mathrm{~Hz}, 2 \mathrm{H}), 7.44-7.36(\mathrm{~m}, 6 \mathrm{H}), 7.32(\mathrm{~d}, J=8.5 \mathrm{~Hz}, 2 \mathrm{H}), 2.76(\mathrm{~d}, J=9.5 \mathrm{~Hz}, 2 \mathrm{H})$, 6.88-6.84 (m, 4H), $4.66(\mathrm{~A}$ of $\mathrm{ABq}, J=12.0 \mathrm{~Hz}, 1 \mathrm{H}), 4.63(\mathrm{~B}$ of $\mathrm{ABq}, J=12.0 \mathrm{~Hz}, 1 \mathrm{H}), 4.51(\mathrm{~A}$ of $\mathrm{ABq}, J=$ $12.0 \mathrm{~Hz}, 1 \mathrm{H}), 4.44(B$ of $A B q, J=12 \mathrm{~Hz}, 1 \mathrm{H}), 4.26(\mathrm{t}, 8.0 \mathrm{~Hz}, 1 \mathrm{H}), 4.07-4.01(\mathrm{~m}, 3 \mathrm{H}), 3.97-3.86(\mathrm{~m}, 4 \mathrm{H})$, 3.80 (s, 3H), 3.70 (s, 3H), 3.73 (dd, J = 11.5, 3.5 Hz, 1H), 3.65 (app t, J = 8.0 Hz, 2 H), 1.92 (q, J = 12 Hz, $1 \mathrm{H}), 1.59$ (br d, $J=12 \mathrm{~Hz}, 1 \mathrm{H}), 1.41(\mathrm{~s}, 3 \mathrm{H}), 1.33(\mathrm{~s}, 6 \mathrm{H}), 1.29(\mathrm{~s}, 3 \mathrm{H}), 1.06(\mathrm{~s}, 9 \mathrm{H}) ;{ }^{13} \mathrm{C}$ NMR $(125 \mathrm{MHz}$, $\left.\mathrm{CDCl}_{3}\right) \mathrm{1}$ 159.1, 159.0, 135.7, 135.6, 133.4, 133.1, 130.7, 130.3, 129.7, 129.6, 129.5, 129.2, 127.7, 127.6, 113.7, 113.6, 109.5, 109.4, 81.0, 77.8, 77.2, 74.0, 72.7, 72.4, 71.5, 71.3, 69.6, 65.3, 63.4, 55.22, 55.20, 27.3, 27.2, 27.0, 26.9, 26.3, 25.1, 19.2; IR (thin film) 2932, $1248 \mathrm{~cm}^{-1}$; HRMS (ESI) calcd for $\mathrm{C}_{48} \mathrm{H}_{62} \mathrm{O}_{10} \mathrm{SiNa} 849.4010 \mathrm{~m} / \mathrm{z}(\mathrm{M}+\mathrm{Na})^{+}$; observed $849.4017 \mathrm{~m} / \mathrm{z}$.

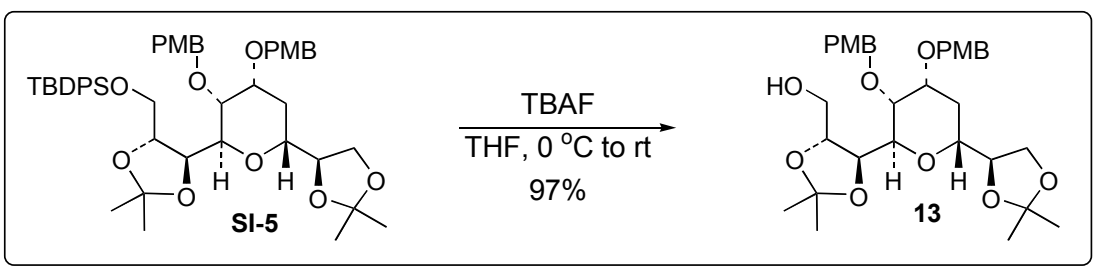

$\{(4 R, 5 R)-5-[(2 R, 3 R, 4 R, 6 R)-6-((R)-2,2-D i m e t h y l-[1,3]$ dioxolan-4-yl)-3,4-bis-(4-

methoxybenzyloxy)-tetrahydropyran-2-yl]-2,2-dimethyl-[1,3]dioxolan-4-yl\}-methanol (13). To a solution of TBDPS ether SI-5 $(230 \mathrm{mg}, 0.28 \mathrm{mmol})$ in THF $(3 \mathrm{~mL})$ at $0{ }^{\circ} \mathrm{C}$ was added a solution of TBAF $(340 \mu \mathrm{L}, 0.34 \mathrm{mmol}, 1 \mathrm{M}$ in THF). The reaction mixture was allowed to warm to ambient temperature, stirred for $18 \mathrm{~h}$ and then diluted with EtOAc. The solution was washed with $1 \mathrm{M} \mathrm{HCl}(3 \mathrm{x})$, sat. $\mathrm{NaHCO}_{3}$, and brine, dried over $\mathrm{Na}_{2} \mathrm{SO}_{4}$, filtered and concentrated in vacuo. The resulting oil was purified by flash column chromatography ( $60 \%$ to $80 \%$ EtOAc-hexane) to give the title compound as a white foam (160 
mg, 97\%): $[\square]_{\mathrm{D}}{ }^{27}=+2.81\left(c 9.7, \mathrm{CHCl}_{3}\right) ;{ }^{1} \mathrm{H} \mathrm{NMR}\left(500 \mathrm{MHz}, \mathrm{CDCl}_{3}\right) \square 7.32$ (d, J = 8.5 Hz, 2H), 7.23 (d, J $=8.5 \mathrm{~Hz}, 2 \mathrm{H}), 6.88-6.84(\mathrm{~m}, 4 \mathrm{H}), 4.67(\mathrm{~A}$ of $\mathrm{ABq}, J=12.0 \mathrm{~Hz}, 1 \mathrm{H}), 4.64(\mathrm{~B}$ of $\mathrm{ABq}, J=12.0 \mathrm{~Hz}, 1 \mathrm{H}), 4.47$ ( $A$ of $A B q, J=11.5 \mathrm{~Hz}, 1 \mathrm{H}), 4.38(B$ of $A B q, J=11.5 \mathrm{~Hz}, 1 \mathrm{H}), 4.12(\mathrm{q}, J=8.0 \mathrm{~Hz}, 1 \mathrm{H}), 4.06-3.98(\mathrm{~m}, 3 \mathrm{H})$, $3.94(\mathrm{dt}, J=7.0,3.5 \mathrm{~Hz}, 1 \mathrm{H}), 3.84-3.82(\mathrm{~m}, 4 \mathrm{H}), 3.80(\mathrm{~s}, 3 \mathrm{H}), 3.79(\mathrm{~s}, 3 \mathrm{H}), 3.68-3.63(\mathrm{~m}, 1 \mathrm{H}), 3.61(\mathrm{t}, J=$ $8 \mathrm{~Hz}, 1 \mathrm{H}$ ), 2.36 (t, $J=6.5 \mathrm{~Hz}, 1 \mathrm{H}), 1.91$ (q, $J=12 \mathrm{~Hz}, 1 \mathrm{H}), 1.50$ (br d, J = $12 \mathrm{~Hz}, 1 \mathrm{H}), 1.41(\mathrm{~s}, 3 \mathrm{H}), 1.39$ (s, 3H), 1.35 (s, 3H), 1.34 (s, 3H); ${ }^{13} \mathrm{C}$ NMR (125 MHz, $\left.\mathrm{CDCl}_{3}\right) \square 159.1,159.0,130.5,130.0,129.5,129.2$, 113.7, 113.5, 109.7, 109.0, 82.1, 78.1, 78.0, 77.2, 73.6, 72.7, 72.0, 71.6, 70.8, 69.4, 65.5, 61.4, 55.1, 27.6, 26.9, 26.8, 26.2, 25.2; IR (thin film) 3496, 2934, $1248 \mathrm{~cm}^{-1}$; HRMS (ESI) calcd for $\mathrm{C}_{32} \mathrm{H}_{44} \mathrm{O}_{10} \mathrm{Na}$ $611.2832 \mathrm{~m} / \mathrm{z}(\mathrm{M}+\mathrm{Na})^{+}$; observed $611.2848 \mathrm{~m} / \mathrm{z}$.

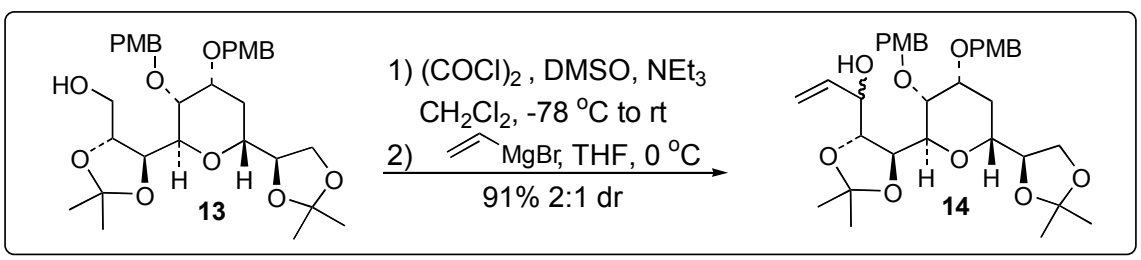

$1-\{(4 R, 5 S)-5-[(2 R, 3 R, 4 R, 6 R)-6-((R)-2,2-D i m e t h y l-[1,3]$ dioxolan-4-yl)-3,4-bis-(4-

methoxybenzyloxy)-tetrahydropyran-2-yl]-2,2-dimethyl-[1,3]dioxolan-4-yl\}-prop-2-en-1-ol (14). To a $-78{ }^{\circ} \mathrm{C}$ solution of distilled oxalyl chloride $(113 \mu \mathrm{L}, 1.32 \mathrm{mmol})$ in $\mathrm{CH}_{2} \mathrm{Cl}_{2}(0.5 \mathrm{~mL})$ was added dropwise a solution of dimethyl sulfoxide $(187 \mu \mathrm{L}, 2.64 \mathrm{mmol})$ in $\mathrm{CH}_{2} \mathrm{Cl}_{2}(0.5 \mathrm{~mL})$. The resulting solution was stirred for $5 \mathrm{~min}$ at $-78{ }^{\circ} \mathrm{C}$. A solution of alcohol $13(710 \mathrm{mg}, 1.2 \mathrm{mmol})$ in $\mathrm{CH}_{2} \mathrm{Cl}_{2}(1 \mathrm{~mL})$ was added and the reaction mixture was allowed to stir $15 \mathrm{~min}$ at $-78{ }^{\circ} \mathrm{C}$. Triethylamine $(0.50 \mathrm{~mL}, 3.6 \mathrm{mmol})$ was added, the reaction was warmed to ambient temperature. The reaction mixture was concentrated in vacuo and the resulting white solid was suspended in $\mathrm{Et}_{2} \mathrm{O}$, filtered though Celite, and concentrated in vacuo to give a yellow oil. DMSO was removed from the resulting oil by Kugelrohr distillation at $\left(50{ }^{\circ} \mathrm{C}, 1.5 \mathrm{mmHg}\right)$ to provide the crude aldehyde which was used directly in the subsequent reaction. $[\square]_{D}{ }^{27}=+1.3$ (c 22.0, $\left.\mathrm{CHCl}_{3}\right) ;{ }^{1} \mathrm{H}$ NMR $\left(500 \mathrm{MHz}, \mathrm{CDCl}_{3}\right) \square 9.81(\mathrm{~s}, 1 \mathrm{H}), 7.31(\mathrm{~d}, J=8.5 \mathrm{~Hz}, 2 \mathrm{H}), 7.24(\mathrm{~d}, 8.5 \mathrm{~Hz}, 2 \mathrm{H})$, 6.88-6.85 (m, 4H), $4.66(\mathrm{~A}$ of $\mathrm{ABq}, 12 \mathrm{~Hz}, 1 \mathrm{H}), 4.63(\mathrm{~B}$ of $\mathrm{ABq}, 12 \mathrm{~Hz}, 1 \mathrm{H}), 4.50-4.42(\mathrm{~m}, 3 \mathrm{H}), 4.30(\mathrm{dd}, J$ $=8.5,6.0 \mathrm{~Hz}, 1 \mathrm{H}), 4.19(\mathrm{q}, J=7 \mathrm{~Hz}, 1 \mathrm{H}), 4.03-3.98(\mathrm{~m}, 2 \mathrm{H}), 3.90(\mathrm{br} \mathrm{s}, 1 \mathrm{H}), 3.85-3.80(\mathrm{~m}, 7 \mathrm{H})$, 3.74-3.68 (m, 2H), 1.93 (q, $12 \mathrm{~Hz}, 1 \mathrm{H}), 1.63$ (br d, $12.5 \mathrm{~Hz}, 1 \mathrm{H}), 1.42(\mathrm{~s}, 3 \mathrm{H}), 1.39$ (s, 3H), 1.35 (s, 6H); ${ }^{13} \mathrm{C}$ NMR $\left(125 \mathrm{MHz}, \mathrm{CDCl}_{3}\right) \square 200.0,159.1,130.3,130.1,129.5,129.0,128.2,113.7,113.6,111.9,109.6$, 83.7, 77.2, 76.7, 74.7, 73.3, 72.3, 71.5, 70.9, 69.8, 55.2, 27.6, 26.9, 26.3, 26.1, 25.3; IR (thin film) 2935, 1513, $1248 \mathrm{~cm}^{-1}$; HRMS (ESI) calcd for $\mathrm{C}_{32} \mathrm{H}_{42} \mathrm{O}_{10} \mathrm{Na} 609.2676 \mathrm{~m} / \mathrm{z}(\mathrm{M}+\mathrm{Na})^{+}$; observed $609.2678 \mathrm{~m} / \mathrm{z}$.

A $0{ }^{\circ} \mathrm{C}$ solution of the crude aldehyde $(1.2 \mathrm{mmol})$ in THF $(3 \mathrm{~mL})$ was treated with vinyl magnesium bromide $\left(2.4 \mathrm{~mL}, 2.4 \mathrm{mmol}, 1 \mathrm{M}\right.$ in THF). The reaction mixture was stirred $15 \mathrm{~min}$ at $0{ }^{\circ} \mathrm{C}$, diluted with sat. $\mathrm{NH}_{4} \mathrm{Cl}$ and EtOAc. The aqueous phase was extracted with EtOAc (2x), and the combine organic extracts were washed with brine, dried over $\mathrm{Na}_{2} \mathrm{SO}_{4}$, filtered and concentrated in vacuo. The resulting oil was purified by flash column chromatography ( $45 \%$ EtOAc-hexane) to give the title compound as a white foam (668 mg, 91\%, 2:1 dr): ${ }^{1} \mathrm{H}$ NMR $\left(500 \mathrm{MHz}, \mathrm{CDCl}_{3}\right) \square 7.31$ (d, J = 8.0 Hz, 2H), 7.24 (d, J = 8.0 Hz, 2H), 
$6.86(\mathrm{t}, J=8.5 \mathrm{~Hz}, 4 \mathrm{H}), 6.08-5.95(\mathrm{~m}, 1 \mathrm{H}), 5.41-5.36(\mathrm{~m}, 1 \mathrm{H}), 5.27-5.22(\mathrm{~m}, 1 \mathrm{H}), 4.64(\mathrm{~s}, 2 \mathrm{H}), 4.49-4.39$ $(\mathrm{m}, 3 \mathrm{H}), 4.17-4.08(\mathrm{~m}, 2 \mathrm{H}), 4.05(\mathrm{~d}, 10.0 \mathrm{~Hz}, 1 \mathrm{H}), 4.01-3.97(\mathrm{~m}, 3 \mathrm{H}), 3.90-3.78(\mathrm{~m}, 7 \mathrm{H}), 3.70-3.68(\mathrm{~m}$, $1 \mathrm{H}), 3.61(\mathrm{q}, J=7.5 \mathrm{~Hz}, 1 \mathrm{H}), 2.60(\mathrm{br} \mathrm{s}, 0.37 \mathrm{H}), 2.32(\mathrm{br} \mathrm{s}, 0.63), 1.96-1.88(\mathrm{~m}, 1 \mathrm{H}), 1.53-1.50(\mathrm{~m}, 1 \mathrm{H})$, $1.43-1.30(\mathrm{~m}, 12 \mathrm{H}) ;{ }^{13} \mathrm{C}$ NMR $\left(125 \mathrm{MHz}, \mathrm{CDCl}_{3}\right) \square 159.1,159.0,137.9,137.3,130.6,130.5,130.2,130.1$, 129.52, 129.46, 129.14, 129.07, 116.2, 115.8, 113.7, 113.58, 113.56, 109.9, 109.72, 109.66, 109.4, 83.9, 83.8, 78.2, 78.1, 78.0, 77.8, 77.2, 75.6, 73.5, 73.3, 73.0, 72.6, 72.4, 72.2, 71.5, 70.9, 70.7, 70. 3, 69.6, $69.5,65.5,55.2,27.6,27.4,27.3,27.14,27.09,26.9,26.4,26.3,25.3,25.2$; IR (thin film) 3479, 2934, $1248 \mathrm{~cm}^{-1}$; HRMS (ESI) calcd for $\mathrm{C}_{34} \mathrm{H}_{46} \mathrm{O}_{10} \mathrm{Na} 637.2989 \mathrm{~m} / \mathrm{z}(\mathrm{M}+\mathrm{Na})^{+}$; observed $637.3004 \mathrm{~m} / \mathrm{z}$.

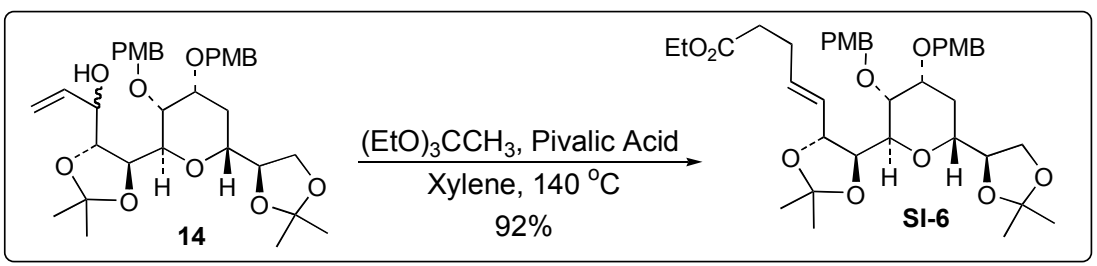

$(E)-5-\{(4 R, 5 R)-5-[(2 R, 3 R, 4 R, 6 R)-6-((R)-2,2-D i m e t h y l-[1,3]$ dioxolan-4-yl)-3,4-bis-(4-

methoxybenzyloxy)-tetrahydropyran-2-yl]-2,2-dimethyl-[1,3]dioxolan-4-yl\}-pent-4-enoic acid ethyl ester (SI-6). A round bottom flask equipped with a short path distillation head and collection flask was charged with allylic alcohol $14(660 \mathrm{mg}, 1.07 \mathrm{mmol})$, triethyl orthoacetate $(1.85 \mathrm{~mL}, 10.7 \mathrm{mmol})$, pivalic acid $(5 \mathrm{mg}, 50 \mu \mathrm{mol})$, and xylene $(8.5 \mathrm{~mL})$. This mixture was heated to $140{ }^{\circ} \mathrm{C}$ for $4 \mathrm{~d}$. Upon completion of the reaction by TLC analysis ( $4 \mathrm{~d}$ ), the mixture was diluted with EtOAc and $1 \mathrm{M} \mathrm{HCl}$, stirred for $15 \mathrm{~min}$, and the organic phase was washed with $1 \mathrm{M} \mathrm{HCl}(2 \mathrm{x})$, sat. $\mathrm{NaHCO}_{3}$, and brine; dried over $\mathrm{Na}_{2} \mathrm{SO}_{4}$, filtered and concentrated in vacuo. The resulting oil was purified by flash column chromatography $(35 \%$ EtOAc-hexane) to give the title compound $(674 \mathrm{mg}, 92 \%)$ : [0] ${ }_{\mathrm{D}}{ }^{27}=+1.5\left(\mathrm{c} \mathrm{7.7}, \mathrm{CHCl}_{3}\right) ;{ }^{1} \mathrm{H}$ NMR $(500$ $\left.\mathrm{MHz}, \mathrm{CDCl}_{3}\right) \square 7.31(\mathrm{~d}, J=8.5 \mathrm{~Hz}, 2 \mathrm{H}), 7.24(\mathrm{~d}, J=8.5 \mathrm{~Hz}, 2 \mathrm{H}), 6.88-6.86(\mathrm{~m}, 4 \mathrm{H}), 5.72$ (dt, $J=15.5,5.5$ $\mathrm{Hz}, 1 \mathrm{H}$ ), $5.52(\mathrm{dd}, J=15.5,7.0 \mathrm{~Hz}, 1 \mathrm{H}$ ), 4.67 (A of ABq, $J=12.5 \mathrm{~Hz}, 1 \mathrm{H}), 4.62(\mathrm{~B}$ of ABq, $J=12.0 \mathrm{~Hz}$, $1 \mathrm{H}), 4.48(\mathrm{~A}$ of $\mathrm{ABq}, J=11.5,1 \mathrm{H}), 4.43(\mathrm{~B}$ of $\mathrm{ABq}, J=12.0 \mathrm{~Hz}, 1 \mathrm{H}), 4.15-4.07(\mathrm{~m}, 4 \mathrm{H}), 3.99-3.95(\mathrm{~m}$, 2H), $3.92(\mathrm{dt}, J=11.5,3.0 \mathrm{~Hz}, 1 \mathrm{H}), 3.86(\mathrm{br} \mathrm{s}, 1 \mathrm{H}), 3.83(\mathrm{dd}, J=8.5,6.5 \mathrm{~Hz}, 1 \mathrm{H}), 3.81(\mathrm{~s}, 3 \mathrm{H}), 3.80(\mathrm{~s}$, $3 \mathrm{H}), 3.75-3.68(\mathrm{~m}, 2 \mathrm{H}), 2.40-2.34(\mathrm{~m}, 4 \mathrm{H}), 1.90(\mathrm{q}, J=12.0 \mathrm{~Hz}, 1 \mathrm{H}), 1.61(\mathrm{br} \mathrm{d}, J=12.5 \mathrm{~Hz}, 1 \mathrm{H}), 1.42$ $(\mathrm{s}, 3 \mathrm{H}), 1.36(\mathrm{~s}, 3 \mathrm{H}), 1.35(\mathrm{~s}, 3 \mathrm{H}), 1.29(\mathrm{~s}, 3 \mathrm{H}), 1.25(\mathrm{t}, J=7.0 \mathrm{~Hz}, 3 \mathrm{H}) ;{ }^{13} \mathrm{C}$ NMR $\left(125 \mathrm{MHz}, \mathrm{CDCl}_{3}\right) \square$ 172.7, 159.12, 159.10, 133.2, 130.6, 130.4, 129.5, 129.0, 128.1, 113.72, 113.65, 109.5, 109.3, 80.6, 79.5, $77.4,76.9,73.0,72.7,71.7,70.9,69.6,65.3,60.3,55.21,55.19,33.5,27.5,27.3,26.89,26.88,26.5$, 25.2, 14.2; IR (thin film) 2934, 1733, 1513, $1248 \mathrm{~cm}^{-1}$; HRMS (ESI) calcd for $\mathrm{C}_{38} \mathrm{H}_{52} \mathrm{O}_{11} \mathrm{Na} 707.3407 \mathrm{~m} / \mathrm{z}$ $(\mathrm{M}+\mathrm{Na})^{+}$; observed $707.3412 \mathrm{~m} / \mathrm{z}$. 


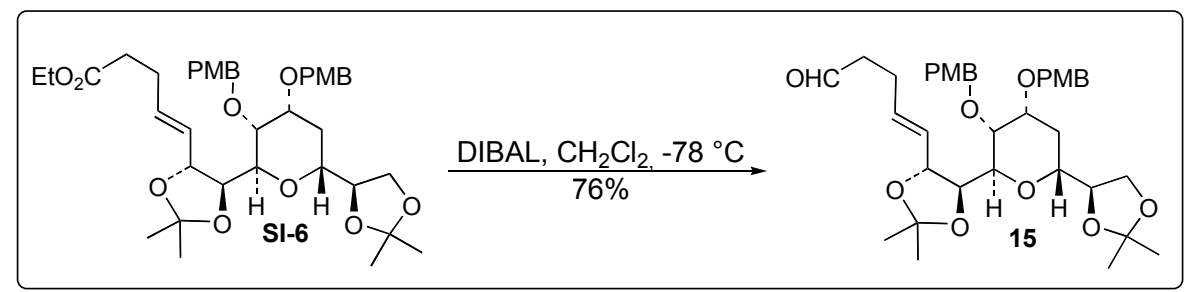

$(E)-5-\{(4 R, 5 R)-5-[(2 R, 3 R, 4 R, 6 R)-6-((R)-2,2-D i m e t h y l-[1,3]$ dioxolan-4-yl)-3,4-bis-(4-methoxybenzyloxy)-tetrahydropyran-2-yl]-2,2-dimethyl-[1,3]dioxolan-4-yl\}-pent-4-enal (15). To a $-78{ }^{\circ} \mathrm{C}$ solution of ester SI-6 (104 mg, $0.15 \mathrm{mmol})$ in $\mathrm{CH}_{2} \mathrm{Cl}_{2}(0.5 \mathrm{~mL})$ was added dropwise DIBAL (150 $\mu \mathrm{L}, 0.15$ $\mathrm{mmol}, 1 \mathrm{M}$ in toluene). The reaction was allowed to stir for $1 \mathrm{~h}$ at $-78{ }^{\circ} \mathrm{C}$, then EtOAc $(200 \mu \mathrm{L})$ was added. The mixture was stirred an additional $10 \mathrm{~min}$, and then diluted with saturated sodium potassium tartrate solution and allowed to warm to ambient temperature and stirred overnight. The biphasic mixture was diluted with $\mathrm{CH}_{2} \mathrm{Cl}_{2}$, and the aqueous phase was extracted with $\mathrm{CH}_{2} \mathrm{Cl}_{2}$ (2x). The combine organic extracts were washed with brine, dried over $\mathrm{Na}_{2} \mathrm{SO}_{4}$, filtered and concentrated in vacuo. The resulting oil was purified by flash column chromatography ( $45 \%$ EtOAc-hexane) to give the title compound as a clear

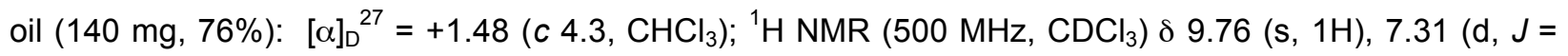
$8.5 \mathrm{~Hz}, 2 \mathrm{H}), 7.24(\mathrm{~d}, J=8.5 \mathrm{~Hz}, 2 \mathrm{H}), 6.87-6.85(\mathrm{~m}, 4 \mathrm{H}), 5.69(\mathrm{dt}, J=15.0,6.5 \mathrm{~Hz}, 1 \mathrm{H}), 5.51(\mathrm{dd}, J=$ $15.0,7.0 \mathrm{~Hz}, 1 \mathrm{H}), 4.67(\mathrm{~A}$ of $\mathrm{ABq}, J=12 \mathrm{~Hz}, 1 \mathrm{H}), 4.61(\mathrm{~B}$ of $\mathrm{ABq}, J=12 \mathrm{~Hz}, 1 \mathrm{H}), 4.48(\mathrm{~A}$ of $\mathrm{ABq}, J=$ $12.0 \mathrm{~Hz}, 1 \mathrm{H}), 4.43(\mathrm{~B}$ of $\mathrm{ABq}, J=11.5 \mathrm{~Hz}, 1 \mathrm{H}), 4.12(\mathrm{q}, J=7 \mathrm{~Hz}, 1 \mathrm{H}), 4.06(\mathrm{t}, J=7.5 \mathrm{~Hz}, 1 \mathrm{H}), 3.99-3.89$ (m, 3H), 3.85-3.79 (m, 8H), 3.74-3.67 (m, 2H), 2.53 (t, $J=7.5 \mathrm{~Hz}, 2 \mathrm{H}), 2.35$ (q, $J=7 \mathrm{~Hz}, 2 \mathrm{H}), 1.88$ (q, $J$ $=12 \mathrm{~Hz}, 1 \mathrm{H}), 1.60(\mathrm{br} \mathrm{d}, J=12.5 \mathrm{~Hz}, 1 \mathrm{H}), 1.41(\mathrm{~s}, 3 \mathrm{H}), 1.35(\mathrm{~s}, 6 \mathrm{H}), 1.28(\mathrm{~s}, 3 \mathrm{H}) ;{ }^{13} \mathrm{C}$ NMR $(125 \mathrm{MHz}$, $\left.\mathrm{CDCl}_{3}\right) \square 201.2,159.04,159.03,132.8,130.5,130.3,129.5,129.0,128.2,113.63,113.57,109.4,109.3$, 80.8, 79.8, 77.7, 77.6, 77.2, 73.3, 73.0, 72.0, 71.2, 69.9, 65.6, 55.13, 55.12, 42.7, 27.3, 26.81, 26.79, 26.4, 25.1, 24.5; IR (thin film) 2933, 1513, $1248 \mathrm{~cm}^{-1}$; HRMS (ESI) calcd for $\mathrm{C}_{36} \mathrm{H}_{48} \mathrm{O}_{10} \mathrm{Na} 663.3145 \mathrm{~m} / \mathrm{z}$ $(\mathrm{M}+\mathrm{Na})^{+}$; observed $663.3148 \mathrm{~m} / \mathrm{z}$.

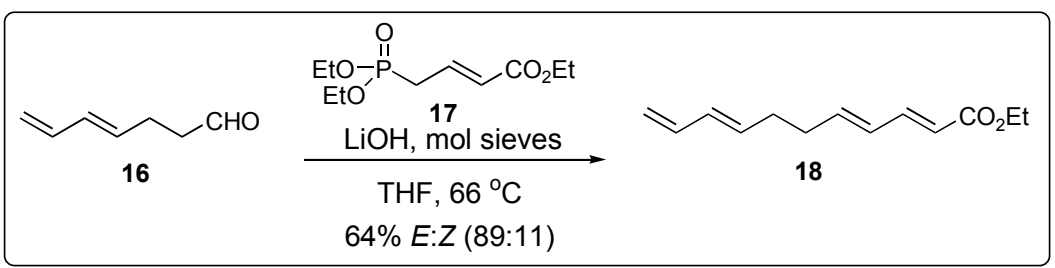

(2E,4E,8E)-Undeca-2,4,8,10-tetraenoic Acid Ethyl Ester (18). To a solution of (E)-hepta-4,6dienal (16) ${ }^{8}$ (2.50 g, $\left.22.7 \mathrm{mmol}\right)$ in THF (150 mL) was added triethyl phosphonocrotonate 17 (5.54 mL, 25 $\mathrm{mmol}), 4 \AA$ molecular sieves $(34.5 \mathrm{~g})$, and $\mathrm{LiOH}(1.05 \mathrm{~g}, 25 \mathrm{mmol})$. The reaction flask was equipped with a reflux condenser and the solution was heated to reflux until with the reaction was judged complete by TLC analysis (2 h). The resulting suspension was filtered through a $\mathrm{SiO}_{2}$ plug and eluted with $\mathrm{Et}_{2} \mathrm{O}$. The filtrate was concentrated in vacuo and purified by flash column chromatography ( $5 \%$ EtOAc-hexane) to give a clear oil $(3.03 \mathrm{~g}, 64 \%, 89: 11 \mathrm{E}: \mathrm{Z})$. An analytical sample was obtained by preparative HPLC (7\% 
EtOAc-hexane). Data for 18: ${ }^{1} \mathrm{H}$ NMR $\left(500 \mathrm{MHz}, \mathrm{CDCl}_{3}\right) \square 7.24(\mathrm{dd}, J=15.0,10.5 \mathrm{~Hz}, 1 \mathrm{H}), 6.29(\mathrm{dt}, J=$ 17.0, $10.0 \mathrm{~Hz}, 1 \mathrm{H}), 6.21-6.04(\mathrm{~m}, 3 \mathrm{H}), 5.79(\mathrm{~d}, J=15.5 \mathrm{~Hz}, 1 \mathrm{H}), 5.67(\mathrm{dt}, J=13.0,5.0 \mathrm{~Hz}, 1 \mathrm{H}), 5.11(\mathrm{~d}, J$ $=17.0 \mathrm{~Hz}, 1 \mathrm{H}), 4.98(\mathrm{~d}, J=10.0 \mathrm{~Hz}, 1 \mathrm{H}), 4.19(\mathrm{q}, J=7.0 \mathrm{~Hz}, 2 \mathrm{H}), 2.28-2.21(\mathrm{~m}, 4 \mathrm{H}), 1.28(\mathrm{t}, J=7.5 \mathrm{~Hz}$, $3 \mathrm{H}) ;{ }^{13} \mathrm{C}$ NMR $\left(125 \mathrm{MHz}, \mathrm{CDCl}_{3}\right) \square 167.1,144.7,143.1,136.9,133.4,131.7,128.8,119.5,115.4,60.1$, 32.4, 31.6, 14.2; IR (thin film) 2981, 1713, $1001 \mathrm{~cm}^{-1}$; HRMS (EI) calcd for $\mathrm{C}_{13} \mathrm{H}_{18} \mathrm{O}_{2} 206.1307 \mathrm{~m} / \mathrm{z} \mathrm{M}^{+}$; observed $206.1304 \mathrm{~m} / \mathrm{z}$.

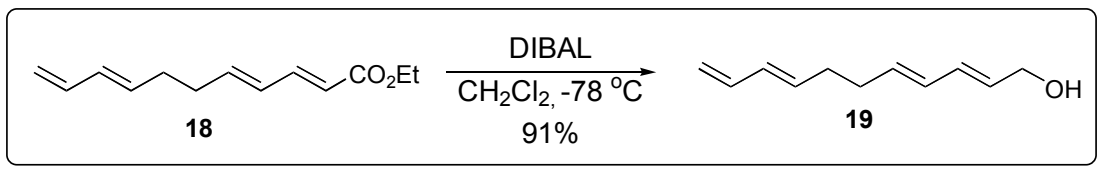

(2E,4E,8E)-Undeca-2,4,8,10-tetraen-1-ol (19). To a $-78{ }^{\circ} \mathrm{C}$ solution of dienoate 18 (0.87 g, 4.2 $\mathrm{mmol})$ in $\mathrm{CH}_{2} \mathrm{Cl}_{2}(18 \mathrm{~mL})$ was added dropwise DIBAL $(12.6 \mathrm{~mL}, 12.6 \mathrm{mmol}, 1 \mathrm{M}$ in toluene). The reaction was stirred for $0.5 \mathrm{~h}$ at $-78{ }^{\circ} \mathrm{C}$, warmed to ambient temperature, quenched with saturated sodium potassium tartrate solution and stirred overnight. The biphasic mixture was separated, the aqueous phase was extracted with EtOAc (2x), and the combine organic extracts were washed with brine, dried over $\mathrm{Na}_{2} \mathrm{SO}_{4}$, filtered and concentrated. The resulting oil was purified by flash column chromatography (15\% EtOAc-hexane) to give a clear oil further enriched in the $E$ olefin isomer (624 $\mathrm{mg}, 91 \%, 95: 5 \mathrm{E:Z}$ ): ${ }^{1} \mathrm{H}$ NMR $\left(500 \mathrm{MHz}, \mathrm{CDCl}_{3}\right) \square 6.31(\mathrm{dt}, J=20.5,10.0 \mathrm{~Hz}, 1 \mathrm{H}), 6.21(\mathrm{dd}, J=15.5,10.5 \mathrm{~Hz}, 1 \mathrm{H}), 6.09-6.04$ (m, 2H), 5.76-5.67 (m, 3H), $5.11(\mathrm{~d}, J=17.0 \mathrm{~Hz}, 1 \mathrm{H}), 4.98(\mathrm{~d}, J=10 \mathrm{~Hz}, 1 \mathrm{H}), 4.15(\mathrm{~d}, J=5.5 \mathrm{~Hz}, 2 \mathrm{H})$, 2.20-2.19 (m, 4H), 2.02 (br s, 1H); ${ }^{13} \mathrm{C}$ NMR (125 MHz, $\left.\mathrm{CDCl}_{3}\right) \square$ 137.0, 134.2, 134.1, 131.6, 131.3, $129.9,129.8,115.1,63.2,32.2,32.1$; IR (thin film) 3325, 2920, $988 \mathrm{~cm}^{-1}$; Anal calcd for $\mathrm{C}_{11} \mathrm{H}_{16} \mathrm{O}: \mathrm{C}$, 80.44; $\mathrm{H}, 9.82$. Found: C, 80.30; H, 9.78.

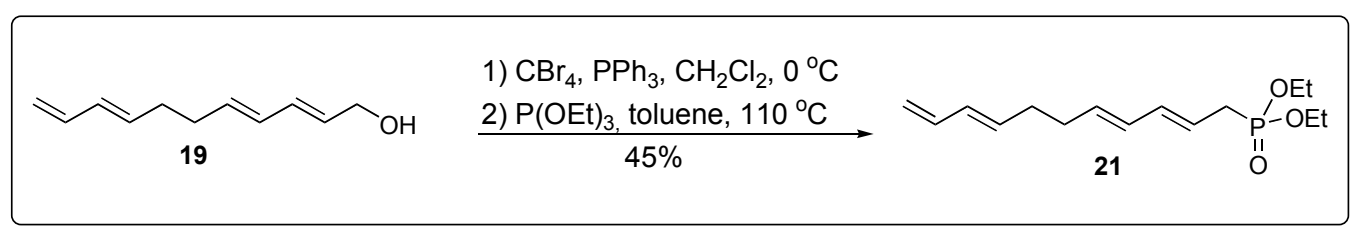

((2E,4E,8E)-Undeca-2,4,8,10-tetraenyl)-phosphonic Acid Diethyl Ester (21). To a solution of $\mathrm{CBr}_{4}(1.01 \mathrm{~g}, 3.0 \mathrm{mmol})$ and $\mathrm{PPh}_{3}(1.03 \mathrm{~g}, 3.95 \mathrm{mmol})$ in $\mathrm{CH}_{2} \mathrm{Cl}_{2}(2 \mathrm{~mL})$ at $0{ }^{\circ} \mathrm{C}$ was added a solution of alcohol 19 (186 mg, $1.13 \mathrm{mmol})$ in $\mathrm{CH}_{2} \mathrm{Cl}_{2}(3 \mathrm{~mL})$. The reaction was stirred for $10 \mathrm{~min}$, filtered through a $\mathrm{SiO}_{2}$ plug (5\% EtOAc-hexane), and the filtrate was concentrated in vacuo. The resulting dienylic bromide was dissolved in toluene $(2.3 \mathrm{~mL})$, treated with triethyl phosphite $(2.3 \mathrm{~mL})$, and the reaction flask was equipped with reflux condenser. The solution was heated at reflux for $18 \mathrm{~h}$, then the toluene was removed in vacuo, and the excess triethyl phosphite removed by Kugelrohr distillation at $\left(50{ }^{\circ} \mathrm{C}, 1.5 \mathrm{~mm} \mathrm{Hg}\right)$. The resulting oil was purified by flash column chromatography ( $80 \%$ EtOAc-hexane) to give the title compound as a clear oil (210mg, 65\%): ${ }^{1} \mathrm{H}$ NMR $\left(500 \mathrm{MHz}, \mathrm{CDCl}_{3}\right) \square 6.31$ (dt, $\left.J=17.0,10.5 \mathrm{~Hz}, 1 \mathrm{H}\right)$, 6.18-6.03 (m, 3H), 5.73-5.62 (m, 2H), 5.53 (sextet, $J=7.0 \mathrm{~Hz}, 1 \mathrm{H}), 5.11(\mathrm{~d}, J=18.5 \mathrm{~Hz}, 1 \mathrm{H}), 4.98(\mathrm{~d}, J=$ 
$10.0 \mathrm{~Hz}, 1 \mathrm{H}), 4.14-4.08(\mathrm{~m}, 4 \mathrm{H}), 2.62(\mathrm{dd}, J=22.5,8.0 \mathrm{~Hz}, 2 \mathrm{H}), 2.20-2.19(\mathrm{~m}, 4 \mathrm{H}), 1.32(\mathrm{t}, J=7.5 \mathrm{~Hz}$, $6 \mathrm{H}) ;{ }^{13} \mathrm{C}$ NMR $\left(125 \mathrm{MHz}, \mathrm{CDCl}_{3}\right) \square 137.1,135.1(\mathrm{~d}, J=13.1 \mathrm{~Hz}), 134.2,133.5,131.4,130.0,119.7(\mathrm{~d}, J=$ $10.9 \mathrm{~Hz}$ ), 115.0, 61.9, 32.16, 32.14, 16.4; IR (thin film) 2982, 1250, $1027 \mathrm{~cm}^{-1}$; HRMS (ESI) calcd for $\mathrm{C}_{15} \mathrm{H}_{25} \mathrm{O}_{3} \mathrm{PNa} 307.1439 \mathrm{~m} / \mathrm{z}(\mathrm{M}+\mathrm{Na})^{+}$; observed $307.1448 \mathrm{~m} / \mathrm{z}$.
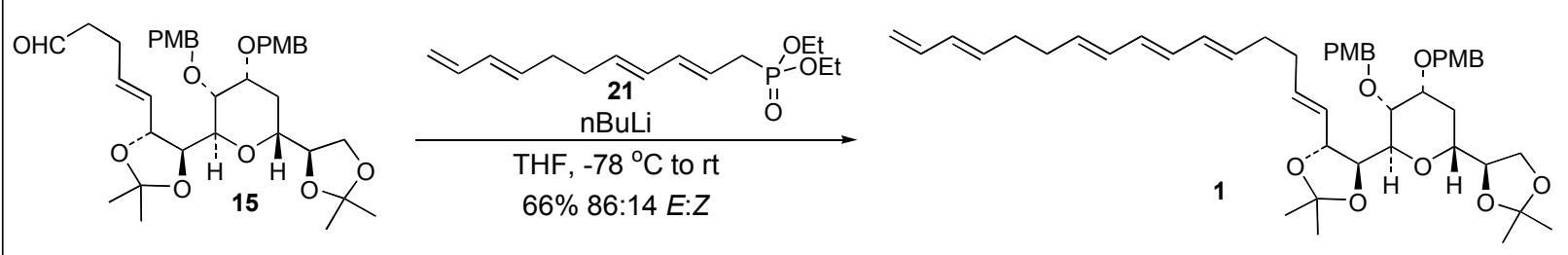

$(2 R, 3 R, 4 R, 6 R)-6-((R)-2,2-D i m e t h y l-[1,3]$ dioxolan-4-yl)-2-[(4R,5R)-5-((1E,5E,7E,9E,13E)hexadeca-1,5,7,9,13,15-hexaenyl)-2,2-dimethyl-[1,3]dioxolan-4-yl]-3,4-bis-(4-methoxybenzyloxy)tetrahydropyran (1). To a $-78{ }^{\circ} \mathrm{C}$ solution of phosphonate 21 (60 mg, $\left.0.21 \mathrm{mmol}\right)$ in THF (400 $\left.\square \mathrm{L}\right)$ was added dropwise n-BuLi ( $82 \mathrm{LL}, 2.4 \mathrm{M}$ in hexanes). The solution was stirred for 20 min at $-78{ }^{\circ} \mathrm{C}$, after which time a solution of aldehyde $15(75 \mathrm{mg}, 0.12 \mathrm{mmol})$ in THF (800 $\mathrm{GL})$ was added dropwise via syringe pump. The reaction mixture was warmed to $-50{ }^{\circ} \mathrm{C}$ and then slowly warmed to ambient temperature. The reaction was stirred for $18 \mathrm{~h}$, diluted with $\mathrm{CH}_{2} \mathrm{Cl}_{2}$ and sat. $\mathrm{NH}_{4} \mathrm{Cl}$, and the aqueaus phase was extrated $(2 x)$ with EtOAc. The combine organic extracts were washed with brine, dried over $\mathrm{Na}_{2} \mathrm{SO}_{4}$, filtered, and concentrated. The resulting crude material was purified by column chormatography (35\% EtOAc:hexane) to give the title compound as a light yellow oil as an $86: 14$ mixture of $E: Z$ isomers (61 mg, 68\%). An analytical sample of 1 was obtained by preparative HPLC (25\% EtOAc:hexane): [D] ${ }^{27}$ $=+1.6$ (c 5.6, $\left.\mathrm{CHCl}_{3}\right) ;{ }^{1} \mathrm{H}$ NMR $\left(500 \mathrm{MHz}, \mathrm{CDCl}_{3}\right) \square 7.31$ (d, J=8.5 Hz, 2H) 7.25 (d, J=9.0 Hz, 2H), 6.88-6.85 (m, 4H), $6.31(\mathrm{dt}, J=17.5,10.0 \mathrm{~Hz}, 1 \mathrm{H}), 6.11-6.04(\mathrm{~m}, 5 \mathrm{H}), 5.74-5.62(\mathrm{~m}, 4 \mathrm{H}), 5.48(\mathrm{dd}, J$ $=15.0,7.0 \mathrm{~Hz}, 1 \mathrm{H}), 5.11(\mathrm{~d}, J=17.0 \mathrm{~Hz}, 1 \mathrm{H}), 4.98(\mathrm{~d}, J=10.0 \mathrm{~Hz}, 1 \mathrm{H}), 4.66(\mathrm{~A}$ of $\mathrm{ABq}, J=12.5 \mathrm{~Hz}, 1 \mathrm{H})$, $4.63(B$ of $A B q, J=12.0 \mathrm{~Hz}, 1 \mathrm{H}), 4.48(\mathrm{~A}$ of $A B q, J=11.5 \mathrm{~Hz}, 1 \mathrm{H}), 4.43(\mathrm{~B}$ of $\mathrm{ABq}, J=11.5 \mathrm{~Hz}, 1 \mathrm{H}), 4.13$ (dd, $J=13.5,6.5 \mathrm{~Hz}, 1 \mathrm{H}), 4.07(\mathrm{t}, J=7.5 \mathrm{~Hz}, 1 \mathrm{H}), 4.00-3.92(\mathrm{~m}, 3 \mathrm{H}), 3.86-3.83(\mathrm{~m}, 2 \mathrm{H}), 3.82(\mathrm{~s}, 3 \mathrm{H})$, 3.81 (s, 3H), 3.74 (dd, $J=8.0,7.0 \mathrm{~Hz}, 1 \mathrm{H}$ ), 3.71 (ddd, $J=11.5,7.0,2.5 \mathrm{~Hz}, 1 \mathrm{H}), 2.20-2.13(\mathrm{~m}, 8 \mathrm{H}), 1.89$ (q, $J=12 \mathrm{~Hz}, 1 \mathrm{H}), 1.62(\mathrm{br} \mathrm{d}, J=12.0 \mathrm{~Hz}, 1 \mathrm{H}), 1.42(\mathrm{~s}, 3 \mathrm{H}), 1.37(\mathrm{~s}, 3 \mathrm{H}), 1.36(\mathrm{~s}, 3 \mathrm{H}), 1.29(\mathrm{~s}, 3 \mathrm{H}) ;{ }^{13} \mathrm{C}$ NMR $\left(125 \mathrm{MHz}, \mathrm{CDCl}_{3}\right) \square$ 159.12, 159.09, 137.1, 134.8, 134.3, 133.5, 133.1, 131.3, 131.1, 131.0, 130.9, $130.8,130.6,130.5,129.5,129.0,127.4,115.0,113.72,113.67,109.5,109.3,80.3,79.7,77.4,76.8$, 72.93, 72.85, 71.6, 70.9, 69.6, 65.3, 55.22, 55.19, 32.4, 32.3, 32.1, 32.0, 27.3, 26.94, 26.90, 26.5, 25.2; IR (thin film) 2931, 1513, $1248 \mathrm{~cm}^{-1}$; Anal calcd for $\mathrm{C}_{47} \mathrm{H}_{62} \mathrm{O}_{9}: \mathrm{C}, 73.22 ; \mathrm{H}, 8.11$. Found: $\mathrm{C}, 73.18 ; \mathrm{H}$, 8.00 .

The olefin geometry of 1 was assigned via HOMO2J and HOMODEC NMR analysis (spectra measured in $d_{6}$-benzene): 


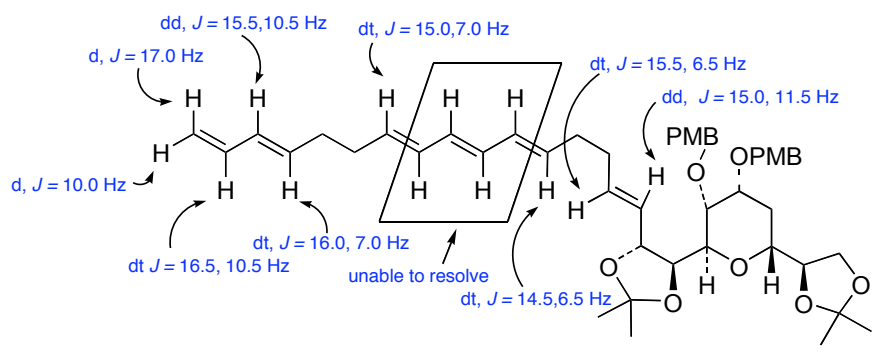

\section{References:}

${ }^{1}$ Ohtami, I.; Kusumi, T.; Kashman, Y.; Kakisawa, H. J. Am. Chem. Soc. 1991, 113, 4092.

${ }^{2}$ Still, W. C.; Kahn, M.; Mitra, A. J. Org. Chem. 1978, 43, 2923.

${ }^{3}$ Flamme, E. M.; Roush, W. R. J. Am. Chem. Soc. 2002, 124, 13644.

${ }^{4}$ Brown, H. C.; Singaram, B. J. Org. Chem. 1984, 49, 945.

${ }^{5}$ Mukai, C.; Moharram, S. M.; Azukizawa, S.; Hanaoka, M. J. Org. Chem. 1997, 62, 8095.

${ }^{6}$ Schmid, C. R.; Bryant, J. D.; Dowlatzedah, M.; Phillips, J. L.; Prather, D. E.; Schantz, R. D.; Sear, N. L.; Vianco, C. S. J. Org. Chem. 1991, 56, 4056.

${ }^{7}$ Wang, L.; Sharpless, K. B. J. Am. Chem. Soc. 1992, 114, 7568.

${ }^{8}$ Hong, S.; Kawaoka, A. M.; Marks, T. J. J. Am. Chem. Soc. 2003, 125, 15878. 